\title{
A New Boundary Harnack Principle (Equations with Right Hand Side)
}

\author{
Mark Allen \& Henrik Shahgholian(i)
}

Communicated by A. Figalli

\begin{abstract}
We introduce a new boundary Harnack principle in Lipschitz domains for equations with a right hand side. Our approach, which uses comparisons and blow-ups, will adapt to more general domains as well as other types of operators. We prove the principle for divergence form elliptic equations with lower order terms including zero order terms. The inclusion of a zero order term appears to be new even in the absence of a right hand side.
\end{abstract}

\section{Introduction}

\subsection{Background}

The well-known boundary Harnack principle states that two non-negative harmonic functions are comparable close to part of the boundary of a given domain, where they both vanish. More exactly, if $u$ and $v$ are harmonic functions in $D \cap B_{1}$ and vanish on $\partial D \cap B_{1}$, with $D$ a Lipschitz domain, then

$$
\frac{1}{C} v(x) \leq u(x) \leq C v(x),
$$

where $C$ depends on space dimension and $u\left(x^{0}\right) / v\left(x^{0}\right)$ for a fixed $x^{0}$ in the domain. ${ }^{1}$

We are interested in extending this result to the case of equations with right hand side. Of course such a general result is doomed to fail, unless some further

${ }^{1}$ The boundary Harnack Principle holds in very general domains, such as NTA domains, and uniform domains. It also holds for solutions to a large class of elliptic equations.

Much of this work was completed while the first author visited KTH Royal Institute of Technology. Shahgholian was supported by Swedish Research Council. 
restrictions are imposed. This can be seen through a simple 2-dimensional example with

$$
u(x)=x_{1} x_{2}\left(x_{1}^{2}-x_{2}^{2}\right), \quad v(x)=x_{2}\left(x_{1}-x_{2}\right)
$$

in the cone $\left\{x_{1}>x_{2}>0\right\}$ with aperture $\pi / 4$, and $\Delta u=0, \Delta v=-2$. Consequently, there cannot exist $C>0$ such that $C u \geq v .^{2}$ Another simple (and discouraging) example is

$$
u(x)=x_{1}^{2}, \quad v(x)=x_{1}
$$

in the set $\left\{x_{1}>0\right\}$. Again, there cannot exist a constant $C$ such that close to the boundary $C u \geq v$.

Two observations can be made from the above examples:

(1) In the first example the domain has a sharp corner at the boundary.

(2) In the second example $\Delta x_{1}^{2}=2$ is both positive and too large.

To put things in perspective, let $D=\left\{x_{1}>0\right\} \cap\left\{x_{1}>x_{2}\right\}$ and consider the following function:

$$
w(x)=x_{1}\left(x_{1}-x_{2}\right) \text { in } D_{r}=D \cap B_{r}(0) .
$$

Now let $u$ be the positive homogeneous harmonic function vanishing on the boundary of the cone $D$, and set $v=u-w$. Since $w>0$ in $D$ (by the definition) we have $u(x)>v(x)$. Next, due to the fact that $w \approx r^{2}$ and $u \approx r^{a}(a<2)$ (for $r$ small), we will have $v>0$. We also have

$$
\Delta u=0, \quad \Delta v=-1 \quad \text { in } D,
$$

with zero boundary values. In particular, we have a boundary Harnack principle $u(x)>v(x)$ where a harmonic function dominates a superharmonic one.

The difference between this example and the first example above is that the cone is wider. The question that naturally arises is: can such behaviour be structured through a general statement, and if so what are the conditions for such a boundary Harnack principle?

Let us also give a simple example where a subsolution controls a solution in the domain $D=\left\{x: x_{1}>0\right\}$. We let $u=x_{1}$ and $v=(\varepsilon / 2) x_{1}^{2}+x_{1}$. Although $v$ is subharmonic, it will control the harmonic solution $u$. In this example $\Delta v$ is small and $v\left(e_{1}\right)$ is large. These are the two conditions that will in general guarantee a subsolution may control a solution.

A further observation is that when the domain $D$ is uniformly $C^{1, D i n i}$ then a boundary Harnack principle holds between a positive harmonic and a superharmonic functions (with bounded right-hand side), vanishing on the boundary $(\partial D) \cap B_{1}$. This is an easy consequence of Hopf's boundary point lemma and

2 One can actually prove the failure of boundary Harnack between harmonic and superharmonic functions, in the first quadrant. This is illustrated in Example 1.2 in [10], in terms of free boundary problems. 
$C^{1}$-regularity of solutions. Indeed, let $u$ be a non-negative harmonic function in $D$ and $v$ satisfy $\Delta v=-1$ in $D$, both with zero Dirichlet data on $(\partial D) \cap B_{1}$. Then by Hopf's boundary principle (applied to $u$ ) and feat that $v$ is uniformly $C^{1}$, we have

$$
\begin{aligned}
& \partial_{v} u \geq C_{0}>0 \quad \text { on } \partial D \cap B_{1}, \quad \text { universal } C_{0}, \\
& \partial_{\nu} v \leq C_{1} \quad \text { on } \partial D, \quad \text { universal } C_{1},
\end{aligned}
$$

where $v$ is the interior normal direction, on $\partial D$. Hence for $k$ large, the function $w_{k}:=k u-v$ satisfies

$$
\partial_{\nu} w_{k}=k \partial_{v} u-\partial_{v} v \geq k C_{0}-C_{1}>0 \quad \text { on } \partial D \cap B_{1} .
$$

Hence $w_{k}>0$ in $D$ in a small neighbourhood of the $\partial D \cap B_{1}$ and inside $D$; this is exactly the boundary Harnack we asked for.

\subsection{Main Result}

Our main result is the following:

Theorem 1.1. Let $D$ be a Lipschitz domain with small Lipschitz constant $c \leq c(n)$ and the origin $0 \in \partial D$. There exists $\varepsilon$ such that if $u$ satisfies

$$
\begin{aligned}
-C_{0} & \leq \Delta u \leq \varepsilon \text { in } D_{L} \cap B_{2}, \\
u & =0 \text { on } \partial D_{L} \cap B_{2}, \\
u & \geq 0 \text { in } D_{L} \cap B_{2}, \\
u\left(e_{n} / 2\right)=1, &
\end{aligned}
$$

and $v$ satisfies

$$
\begin{aligned}
|\Delta v| & \leq C_{0} \text { in } D_{L} \cap B_{2}, \\
v & =0 \text { on } \partial D_{L} \cap B_{2}, \\
\sup _{B_{1} \cap D_{L}}|v| & =1,
\end{aligned}
$$

then for a universal positive constants $C, \beta>$, depending on $n, C_{0}$, one has

$$
\left\|\frac{v}{u}\right\|_{C^{0, \beta}\left(B_{1 / 4}\right)} \leq C
$$

Remark 1.2. We prove Theorem 1.1 for the Laplace operator, however the result will also be true for divergence form operators as given in Theorem 4.7. The methods in Lemmas 3.12-3.14 will also apply to divergence form operators to show that the quotient is not only bounded as stated in Theorem 4.7 but also Hölder continuous up to the boundary. 
For instructional reasons and for the benefit of the non-expert reader, we first state and prove the theorem inside a cone; see Theorem 2.1. The proof in this case presents the main ideas for the general case. Next, we do the same for a Lipschitz domain; see Theorem 3.10. We then apply the ideas to divergence operators, see Theorem 4.7. Our result holds even when including zero-order terms. This result appears to be new even when considering the standard boundary Harnack principle without right hand side, that is for solutions rather than supersolutions.

In proving our boundary Harnack principle, we will often utilize the boundary Harnack principle (without right hand side) for two nonnegative solutions. To avoid confusion we will henceforth refer to this as the "standard boundary Harnack principle".

\subsection{Related Results}

As previously mentioned the boundary Harnack principle has been proven in very general domains as well as for various operators. Recently, De Silva and Savin in [5] generalized the result in a new direction by showing that improving the regularity of the boundary improves the regularity of the boundary Harnack inequality. Their result applies to elliptic operators in divergence form with appropriate smoothness assumptions on the coefficients. Roughly speaking, if $u, v$ vanish on $\partial D$ with $\mathcal{L} u=0$ and $\mathcal{L} v=f$ in $D$, and if $u>0$ in $D$ and $\partial D \in C^{k, \beta}$, then

$$
\left\|\frac{v}{u}\right\|_{C^{k, \beta}} \leq C\left(\|v\|_{L^{\infty}}+\|f\|_{C^{k-1, \beta}}\right) .
$$

The result in [5] illustrates the principle that improving the regularity of the boundary gives a boundary Harnack principle for solutions with a right hand side. Our result shows that Lipschitz regularity is a sufficient condition to obtain an estimate of the form (1.1) when $k=0$, and the allowed behavior for $f$ is determined by the Lipschitz constant.

Another related result is found in [11], where it is shown that a superharmonic function is comparable to the first eigenfunction for a domain in $\mathbb{R}^{2}$ with finitely many corners and with an interior cone condition.

\subsection{Applications}

We present two applications of our boundary Harnack principle: to the HeleShaw flow and to the obstacle problem.

1.4.1. Hele-Shaw Flow The Hele-Shaw flow may be formulated as follows: for $t>0$ we define $u^{t}(x)$ as the solution of

$$
\Delta u^{t}(x)=\chi_{\Omega^{t}}-\chi_{\Omega^{0}}-t \delta_{z} \quad \text { in } \mathbb{R}^{n},
$$

where $\Omega^{0}$ is the initial domain (filled with liquid) and $\Omega^{t}=\left\{u^{t}>0\right\}$, and $z \in \Omega^{0}$ is the liquid-injection point. The Dirac mass at the point $z$ means we inject more fluid at that point. This formulation is obtained after a Baiocchi-transformation, which is easily found in the classical literature for free boundary problems. 
Now suppose we do the Hele-Shaw experiment on a "table" having corners of various angles. More exactly, suppose we consider

$$
\Delta u^{t}(x)=\chi_{\Omega^{t}}-\chi_{\Omega^{0}}-t \delta_{z} \quad \text { in } D, \quad u^{t}(x)=0 \text { on } \partial D,
$$

where $D \supset \Omega^{0}$ is a given domain. The zero boundary data on $\partial D$ means that the liquid, when reaching the edge of the table, will fall to the ground. The question is whether the liquid will reach all points of the boundary of $D$ in finite time $t_{0}<\infty$.

By a barrier argument (see [10]) one can show that in two dimensions corners with angle smaller than or equal to $\pi / 2$ will not get wet; an analogous result for higher dimensions is a consequence of Theorem 2.5 and 2.6 in this paper. Now the question is what can happen when the angle of the corner is larger than $\pi / 2$; will the liquid reach such a corner? The answer to this question is yes; see [10].

We now consider $D$ with Lipschitz boundary with Lipschitz constant $L<1$. As a consequence of Theorem 3.10 we now show that every point of the boundary will get wet. We need to show for large values of $t$ that $u^{t}>0$ in $D \cap B_{r}\left(z^{1}\right)$, for any $z^{1} \in \partial D$. We write $u^{t}(x)=h^{t}(x)-k(x)$ where $h^{t}$ is the harmonic function in $B_{r}\left(z^{1}\right) \cap D$ with boundary values $h^{t}=u^{t}$, and $k$ ( $t$-independent) satisfies

$$
\Delta k=-1 \quad \text { in } B_{r}\left(z^{1}\right) \cap D,
$$

with zero boundary values on $\partial\left(B_{r}\left(z^{1}\right) \cap D\right)$. It suffices then to show that for large values of $t$ we have

$$
h^{t} \geq k \quad \text { in } B_{r}\left(z^{1}\right) \cap D .
$$

By Theorem 3.10, for fixed $t$ there exists some large constant $C_{t}$ such that $C_{t} h^{t} \geq k$. By the standard boundary Harnack principle

$$
\sup _{B_{r / 2}\left(z^{1}\right) \cap D} \frac{h^{t}}{h^{s}} \leq C \frac{h^{t}\left(z^{2}\right)}{h^{s}\left(z^{2}\right)},
$$

for a fixed $z^{2} \in B_{r}\left(z^{1}\right) \cap D$. Since $\lim _{s \rightarrow \infty} h^{s}\left(z^{2}\right)=\infty$, we choose $s$ large enough so that $h^{t}\left(z^{2}\right) / h^{s}\left(z^{2}\right)<C^{-1} / C_{t}$. Then

$$
\sup _{B_{r / 2}\left(z^{1}\right) \cap D} \frac{h^{t}}{h^{s}} \leq C \frac{h^{t}\left(z^{2}\right)}{h^{s}\left(z^{2}\right)} \leq \frac{C C^{-1}}{C_{t}},
$$

so that $h^{s} \geq C_{t} h^{t} \geq k$ to conclude that $u^{t}>0$ in $B_{r / 2}\left(z^{1}\right) \cap D$, for $t$ large enough.

A related question to the Hele-Shaw flow reaching corners, is whether for $D \subset \mathbb{R}^{n}$, with $0 \in \partial D$, and $\partial B_{1} \cap D \neq \emptyset$, a solution to

$$
\Delta v_{m}=1 \quad \text { in } D \cap B_{1}, \quad v_{m}=0 \text { on } \partial D \cap B_{1} \quad v_{m}=m \text { on } \partial B_{1} \cap D,
$$

will be non-negative in $D \cap B_{1}$ for $m$ large enough. The answer to this question is already given in the discussion for Hele-Shaw experiment above. 
1.4.2. Obstacle Problem A further application can be made to the regularity theory of the free boundary for the obstacle problem, that is formulated as a solution to

$$
\Delta v=h \chi_{\{v>0\}}, \quad v \geq 0 \quad \text { in } B_{1},
$$

with $h \geq c_{0}>0$ Lipschitz, and a prescribed Dirichlet data on $\partial B_{1}$. In proving regularity of the free boundary for the obstacle problem one can show that if a free boundary point $z \in \partial\{v>0\} \cap B_{1 / 2}$ is not a cusp point, then for some $r>0$ and direction $e, v_{e}>0$ in the set $\{v>0\} \cap B_{r}(z)$, and that the free boundary is Lipschitz in $B_{r}(z)$.

One can actually show that the Lipschitz norm can be taken as small as one wishes by taking the neighbourhood of $z$ smaller. The proof for (non-uniform) Lipschitz regularity is actually much simpler than proving uniform regularity. We refer to [9] for background and details as well as other related original references.

The boundary Harnack principle in this paper allows us to deduce $C^{1, \alpha}$-regularity of the free boundary for the obstacle problem, in an elementary way. ${ }^{3}$ Indeed, we consider the function $H(x)=v_{e_{1}}-C v$, which satisfies ${ }^{4}$

$$
H>0, \quad v_{e_{1}}>0, \quad \Delta H=h_{e_{1}}-C h \leq 0 \quad \text { in }\{v>0\} \cap B_{r}(z) .
$$

We will now apply Theorem 1.1 to the functions $H=v_{e_{1}}-C v$ and $v_{e}$ (which satisfies $\Delta v_{e}=h_{e}$ ) for any direction $e$ orthogonal to $e_{1}$. Both $H$ and $v_{e}$ vanish on the free boundary $\partial\{v>0\}$. By taking a neighborhood of $z \in \partial\{v>0\} \cap B_{1 / 2}$ small enough, the Lipschitz norm of $\partial\{v>0\} \cap B_{1 / 2}$ can be made arbitrarily small. Since $\Delta H \leq 0$ and $|\Delta H|$ is bounded (due to Lipschitz regularity of $h$ ), we may apply Theorem 1.1 to conclude that

$$
\frac{v_{e}}{v_{e_{1}}-C v}
$$

is $C^{\alpha}$ inside $\{v>0\}$ (close to the free boundary point $z$ ).

Next fix a level surface $v=l$, and denote the free boundary as a Lipschitz graph $x_{1}=G\left(x^{\prime}\right) .{ }^{5}$ Consider $v\left(G\left(x^{\prime}\right), x^{\prime}\right)=l$, which after differentiation in the $e$-direction gives

$$
-G_{e}=\frac{v_{e}}{v_{e_{1}}},
$$

inserting this into (1.2) gives us

$$
\frac{v_{e}}{v_{e_{1}}-C v}=\frac{\frac{v_{e}}{v_{e_{1}}}}{1-\frac{C v}{v_{e_{1}}}}=\frac{-G_{e}}{1-\frac{C l}{v_{e_{1}}}},
$$

3 This was done for constant $h$ in [1] using the standard boundary Harnak principle for Lipschitz domains.

4 Actually this conclusion is part of proving the Lipschitz regularity of the free boundary; see [3]

5 The level surface is smooth since $v_{e_{1}}>0$ there. 
is $C^{\alpha}$, independent of $l$. Since $v_{e_{1}} \approx \sqrt{l}$ we have that

$$
\frac{v_{e}}{v_{e_{1}}-C v}=\frac{-G_{e}}{1-\frac{C l}{v_{e_{1}}}} \rightarrow-G_{e},
$$

as $l \rightarrow 0 .{ }^{6}$ Hence $G_{e}$ is $C^{\alpha}$.

\subsection{Future Directions}

It seems plausible that the results presented in this paper can be generalized to other operators, as well as more complicated domains. Here we have chosen to treat the problem in Lipschitz domains only. In the final section we consider second order elliptic equations of divergence form. The coefficients are variable and assumed to be only bounded and measurable.

Key elements of our approach are the standard boundary Harnack principle, barrier arguments, as well as scaling and blow-up invariance. Since our approach is indirect and uses scalings, the core idea is to look at nonnegative solutions on global domains. The technical difficulties that seem to arise for generaliztions of our result concern the invariance of the domains in scaling.

The methods presented here should also work to prove a boundary Harnack principle for the positivity set of a solution to the thin obstacle problem as long as it is assumed a priori that the free boundary is Lipschitz. Then we may argue in a similar way as above for the thin obstacle problem, with equations having Lipschitz right hand side; see [1] in combination with our result.

\section{Boundary Harnack in Cones}

We let $\mathcal{C}$ be any open cone in $\mathbb{R}^{n}$, with vertex at the origin such that $\mathcal{C} \cap \mathbb{S}^{n-1}$ is connected. If $u$ is any harmonic function on $\mathcal{C}$ with $u=0$ on $\partial \mathcal{C}$, then in spherical coordinates

$$
u(r, \theta)=\sum_{k=1}^{\infty} r^{\alpha_{k}} f_{k}(\theta),
$$

where $f_{k}$ are the eigenfunctions to the Laplace-Beltrami on $\mathcal{C} \cap \partial B_{1}$. If $u$ is harmonic on $\mathcal{C}$ and nonnegative, then $u$ is unique up to multiplicative constant to $r^{\alpha_{1}} f_{1}(\theta)$.

Theorem 2.1. Let $\mathcal{C}$ be a connected open cone in $\mathbb{R}^{n}$ with $\partial \mathcal{C} \cap \mathbb{S}^{n-1}$ an $(n-2)$ dimensional $C^{1, \alpha}$ submanifold. ${ }^{7}$ Let $r^{\alpha_{1}} f_{1}(\theta) \geq 0$ be harmonic in $\mathcal{C}$ with zero Dirichlet boundary data. Let u solve

$$
\begin{aligned}
\Delta u & =0 \text { in } \mathcal{C} \cap B_{1}, \\
u & \geq 0 \text { in } \mathcal{C} \cap B_{1}, \\
u & =0 \text { on } \partial \mathcal{C} \cap B_{1},
\end{aligned}
$$

\footnotetext{
6 See for example [9].

7 The assumption that $\partial \mathcal{C} \cap \mathbb{S}^{n-1}$ is an $(n-2)$-dimensional manifold of class $C^{1, \alpha}$ is not necessary. As we will see in Section $3, \partial \mathcal{C} \cap \mathbb{S}^{n-1}$ may be a Lipshitz manifold provided the Lipschitz constant is small enough depending on $\gamma$, that appears in (2.2).
} 
and let $v$ satisfy

$$
\begin{array}{cl}
0 \geq \Delta v(x) \geq-C_{0}|x|^{\gamma} & \text { in } \mathcal{C} \cap B_{1}, \\
v=0 & \text { on } \partial \mathcal{C} \cap B_{1}, \\
|v| \leq C_{0} & \text { in } \mathcal{C} \cap B_{1},
\end{array}
$$

with $2-\alpha_{1}+\gamma>0$. If $x^{0} \in \mathcal{C} \cap B_{1}$, then there exists a constant $C$ depending only on $\mathcal{C}, 2-\alpha_{1}+\gamma$, dimension $n$, and dist $\left(x^{0}, \partial\left(\mathcal{C} \cap B_{1}\right)\right)$ such that if $u\left(x^{0}\right)=v\left(x^{0}\right)=1$, then

$$
C^{-1} v(x) \leq u(x) \leq C v(x) \text { for any } x \in \mathcal{C} \cap B_{1 / 2} .
$$

Theorem 2.1 is a boundary Harnack principle, but with a right hand side. Clearly, a harmonic solution will control a subsolution. The significance of Theorem 2.1 is that a harmonic solution can control a supersolution, and that the allowed behavior for the right hand side depends on the opening of the cone or more explicitly on $\alpha_{1}$. When the opening of the cone is large (so that $\alpha_{1}$ is small), then negative values for $\gamma$ are allowed, and the right hand side can have singular behavior near the boundary. When the opening of the cone is small (so that $\alpha_{1}$ is large), then $\gamma$ must be positive and large, so that the right hand side must decay as it approaches the boundary.

In order to prove Theorem 2.1, we will need the following convergence result:

Lemma 2.2. Let $\mathcal{C}$ be an open cone with $\partial \mathcal{C} \cap \mathbb{S}^{n-1}$ an $(n-2)$-dimensional $C^{1, \alpha}$ submanifold. Fix $0<\varepsilon<R$ and let $\gamma>\alpha_{1}-2$. For any sequence $v_{k}$ satisfying

$$
\begin{aligned}
\left|\Delta v_{k}\right| & \leq C_{0}|x|^{\gamma} & \text { in } \mathcal{C} \cap B_{R}, \\
\left|v_{k}\right| & \leq C_{0} & \text { in } \mathcal{C} \cap B_{R}, \\
v_{k} & =0 & \text { on } \partial \mathcal{C} \cap B_{R},
\end{aligned}
$$

there exists a subsequence $v_{k} \rightarrow v$ uniformly on $\mathcal{C} \cap B_{R-\varepsilon}$ with $v$ inheriting the above properties.

Proof. Since $\mathcal{C} \cap \partial B_{R}$ is a $C^{1, \alpha}$ Lipschitz submanifold, $\mathcal{C}$ is compactly contained in a slightly larger cone $\mathcal{C}^{\prime} \supset \mathcal{C}$, and the unique nonnegative harmonic solution with zero Dirichlet data on $\partial \mathcal{C}^{\prime}$ is given as $r^{\alpha_{1}-\delta} f_{\delta}$. We note that $f_{\delta}(\theta)$ is uniformly bounded away from 0 on $\mathcal{C}$. We let $h=M_{1} r^{\alpha_{1}-2 \delta} f_{\delta}$ with $M_{1}$ chosen later. Then

$$
\Delta h=M_{1}\left[\left(\alpha_{1}-2 \delta\right)\left(\alpha_{1}-2 \delta+n-2\right)-\left(\alpha_{1}+\delta\right)\left(\alpha_{1}+\delta+n-2\right)\right] r^{\alpha_{1}-2-2 \delta} f_{\delta} .
$$

Since $\alpha_{1}-2-2 \delta<\gamma$, we may choose $M_{1}$ large enough such that

$$
\Delta h \leq-C_{0}|x|^{\alpha_{1}-2-2 \delta} \leq-C_{0}|x|^{\gamma} .
$$

Using $h$ as a barrier, the convergence result will follow by standard techniques.

An alternate proof of the above lemma, for a more general domain and more general operator, is given in the proof of Lemma 4.5.

We now give a proof of the main theorem in this Section. 
Proof of Theorem 2.1. Fix $x^{0} \in \mathcal{C} \cap B_{1 / 2}$, and consider the nonnegative homogeneous solution $u=r^{\alpha_{1}} f_{1}(\theta)$. By the standard boundary Harnack principle, any solution $u$ as given in the statement of Theorem 2.1 will be comparable, and consequently bounded from below up to a multiplicative constant, by $r^{\alpha_{1}} f(\theta)$. Thus, we consider $u=r^{\alpha_{1}} f(\theta)$. Furthermore, any function $v$ as given in the statement of Theorem 2.1 will be bounded from above by a constant multiple of the superharmonic function defined by

$$
\begin{aligned}
\Delta v & =-|x|^{\gamma} & & \text { in } \mathcal{C} \cap B_{1}, \\
v & \geq 0 & & \text { in } \mathcal{C} \cap B_{1}, \\
v & =0 & & \text { on } \partial \mathcal{C} \cap B_{1}, \\
v & =1 & & \text { on } \mathcal{C} \cap \partial B_{1} .
\end{aligned}
$$

Thus, it suffices to prove the theorem for $v$ as defined above and with $u=r^{\alpha_{1}} f(\theta)$.

In the following we use $r$ as a scaling parameter which should not be confused when $r$ is used as the polar axis for homogeneous functions. We first show that there exists some constant $C$ such that $v\left(r x^{0}\right) \leq C u\left(r x^{0}\right)$ for all $0<r \leq 1 / 2$. Suppose, by way of contradiction, that no such $C$ exists, so that if $v\left(t x^{0}\right)=t^{\alpha_{1}} h(t)$, then $\lim \sup _{t \rightarrow 0} h(t)=\infty$. We note that $h$ is continuous away from the origin since $v$ is continuous. For $0<r<1$, we consider the rescaled functions

$$
w_{r}(x):=\frac{v(r x)-\frac{v\left(r x^{0}\right)}{u\left(r x^{0}\right)} u(r x)}{\sup _{B_{1} \cap \mathcal{C}}\left|v(r x)-\frac{v\left(r x^{0}\right)}{u\left(r x^{0}\right)} u(r x)\right|},
$$

defined on $B_{1 / r}$. We point out that $w_{r}\left(x^{0}\right)=0$ and $\sup _{B_{1} \cap \mathcal{C}}\left|w_{r}\right|=1$. We also define

$$
a_{k}:=\sup _{B_{1} \cap \mathcal{C}}\left|v\left(2^{-k} x\right)-\frac{v\left(2^{-k} x^{0}\right)}{u\left(2^{-k} x^{0}\right)} u\left(2^{-k} x\right)\right| 2^{k \alpha_{1}} .
$$

By letting $x=t x^{0}$, we have

$$
v(r x)-\frac{v\left(r x^{0}\right)}{u\left(r x^{0}\right)} u(r x)=[h(r t)-h(r)](r t)^{\alpha_{1}} .
$$

If $r_{k}=2^{-k}$, and if $1 / 2 \leq t \leq 1$, we have

$$
\operatorname{osc}_{\left[r_{k} / 2, r_{k}\right]} h \leq a_{k}
$$

Since $\lim \sup _{t \rightarrow 0} h(t)=+\infty$, it follows that

$$
\sum a_{k}=+\infty
$$

By the definition of $w_{r}$, we have

$$
\sup _{B_{2^{j}}}\left|w_{r_{k}}(x)\right|=\frac{\sup _{B_{2 j}}\left|v\left(r_{k} x\right)-\frac{v\left(r_{k} x^{0}\right)}{u\left(r_{k} x^{0}\right)} u\left(r_{k} x\right)\right|}{a_{k} r_{k}^{\alpha_{1}}} .
$$


We use the triangle inequality to obtain

$$
\begin{aligned}
\left|v\left(r_{k} x\right)-\frac{v\left(r_{k} x^{0}\right)}{u\left(r_{k} x^{0}\right)} u\left(r_{k} x\right)\right| \leq & \left|v\left(r_{k} x\right)-\frac{v\left(2^{j} r_{k} x^{0}\right)}{u\left(2^{j} r_{k} x^{0}\right)} u\left(r_{k} x\right)\right| \\
& +\sum_{i=0}^{j-1}\left|\frac{v\left(2^{j-i} r_{k} x^{0}\right)}{u\left(2^{j-i} r_{k} x^{0}\right)} u\left(r_{k} x\right)-\frac{v\left(2^{j-i-1} r_{k} x^{0}\right)}{u\left(2^{j-i-1} r_{k} x^{0}\right)} u\left(r_{k} x\right)\right| .
\end{aligned}
$$

To bound the first term in the above inequality, we have, by definition, that

$$
\sup _{B_{2^{j}}}\left|v\left(r_{k} x\right)-\frac{v\left(2^{j} r_{k} x^{0}\right)}{u\left(2^{j} r_{k} x^{0}\right)} u\left(r_{k} x\right)\right|=a_{k-j}\left(2^{j} r_{k}\right)^{\alpha_{1}} .
$$

To bound the second term in the inequality, we note first that

$$
\begin{aligned}
& \left|\frac{v\left(2^{j-i} r_{k} x^{0}\right)}{u\left(2^{j-i} r_{k} x^{0}\right)} u\left(2^{j-i-1} r_{k} x^{0}\right)-\frac{v\left(2^{j-i-1} r_{k} x^{0}\right)}{u\left(2^{j-i-1} r_{k} x^{0}\right)} u\left(2^{j-i-1} r_{k} x^{0}\right)\right| \\
& \quad=\left|\frac{v\left(2^{j-i} r_{k} x^{0}\right)}{u\left(2^{j-i} r_{k} x^{0}\right)} u\left(r_{k} 2^{j-i-1} x^{0}\right)-v\left(2^{j-i-1} r_{k} x^{0}\right)\right| \\
& \quad \leq a_{k-(j-i)}\left(2^{j-i} r_{k}\right)^{\alpha_{1}} .
\end{aligned}
$$

Also, we note that there exists $C$ depending on $x^{0}$ such that if $|x|=1$, then $u(r x) \leq C u\left(r x^{0}\right)$. If we utilize the homogeneity of $u$, we conclude that

$$
\sup _{B_{2^{j}}} u\left(r_{k} x\right) \leq C u\left(2^{j} r_{k} x^{0}\right) .
$$

We use this and the homogeneity of $u$ to obtain

$$
\begin{aligned}
\sup _{B_{2^{j}}} \mid & \left|\frac{v\left(2^{j-i} r_{k} x^{0}\right)}{u\left(2^{j-i} r_{k} x^{0}\right)} u\left(r_{k} x\right)-\frac{v\left(2^{j-i-1} r_{k} x^{0}\right)}{u\left(2^{j-i-1} r_{k} x^{0}\right)} u\left(r_{k} x\right)\right| \\
= & \sup _{B_{2^{j}}} \frac{u\left(r_{k} x\right)}{u\left(2^{j-i-1} r_{k} x^{0}\right)} \\
& \times\left|\frac{v\left(2^{j-i} r_{k} x^{0}\right)}{u\left(2^{j-i} r_{k} x^{0}\right)} u\left(2^{j-i-1} r_{k} x^{0}\right)-v\left(2^{j-i-1} r_{k} x^{0}\right)\right| \\
\leq & \frac{C u\left(2^{j} r_{k} x^{0}\right)}{u\left(2^{j-i-1} r_{k} x^{0}\right)} a_{k-(j-i)}\left(2^{j-i} r_{k}\right)^{\alpha_{1}} \\
= & C a_{k-(j-i)}\left(2^{j+1} r_{k}\right)^{\alpha_{1}} .
\end{aligned}
$$

From (2.3) we may apply Lemma A.1 to the sequence $\left\{a_{k}\right\}$ to conclude that there exists a subsequence $k_{l}$ such that for any $j \in \mathbb{N}$,

$$
\limsup _{k_{l} \rightarrow \infty} \frac{\sum_{i=1}^{j} a_{k_{l}-i}}{a_{k_{l}}} \leq j .
$$


By choosing $r_{k_{l}}$ and combining the above inequality with (2.4), (2.5) and (2.6), we conclude that

$$
\sup _{B_{2^{j}}}\left|w_{r_{k_{l}}}(x)\right| \leq C j 2^{j \alpha_{1}}
$$

for $j \leq k_{l}$.

We will now bound the Laplacian of $w_{r_{k}}$. From the choice of $a_{k_{l}}$ in Lemma A.1 and the fact that $\sum a_{k}=+\infty$ it follows that eventually $a_{k_{l}} \geq k_{l}^{-2}$, so that if $r_{k_{l}}=2^{-k_{l}}$, then

$$
a_{k_{l}} \geq\left[\ln \left(1 / r_{k}\right)\right]^{-2} \text {. }
$$

We now use the assumption $2-\alpha_{1}+\gamma>0$. Since $\Delta u=0$ and $\Delta v=-|x|^{\gamma}$ we have that for a sequence $r_{k_{l}} \rightarrow 0$, it holds that

$$
\left|\Delta w_{k}\right| \leq \frac{r_{k}^{2+\gamma}|x|^{\gamma}}{\sup _{B_{1} \cap \mathcal{C}}\left|v(r x)-\frac{v\left(r x^{0}\right)}{u\left(r x^{0}\right)} u(r x)\right|} \leq C r_{k}^{2-\alpha_{1}+\gamma}\left[\ln \left(1 / r_{k}\right)\right]^{2}|x|^{\gamma} \rightarrow 0
$$

as long as $2-\alpha_{1}+\gamma>0$.

By Lemma 2.2 there exists a subsequence $w_{k} \rightarrow w$ uniformly in $B_{R} \cap \mathcal{C}$ for any $R>0$. Furthermore, $w$ will satisfy

$$
\begin{aligned}
& \Delta w=0 \quad \text { from (2.8), } \\
& \sup |w|=1 \text {, } \\
& w\left(x^{0}\right)=0 \\
& \text { (iv) } \quad w(x) \leq C|x|^{\alpha_{1}} \ln (|x|+1) \quad \text { for }|x| \geq 1 \text { from (2.7). }
\end{aligned}
$$

By property (ii) we have that $w$ is not identically zero. By property (iii) we have that $w$ changes sign, so that by (2.1) we have $\sup _{B_{R}}|w| \geq c R^{\alpha_{2}}$ for $R \geq 1$. Since $|x|^{\alpha_{1}} \ln (|x|+1)<|x|^{\alpha_{2}}$, for large $x$, this contradicts property (iv).

Thus, we have shown that for any $x^{0} \in \mathcal{C}$ there exists a constant $C$ depending on $x^{0}$ such that

$$
v\left(r x^{0}\right) \leq C u\left(r x^{0}\right) \text { for any } 0<r \leq 1 / 2 .
$$

For any other point $x^{1} \in \mathcal{C} \cap B_{1 / 2}$, we rescale by $v\left(x\left|x^{1}\right| / 2\right)$, and note that $\left|\Delta v\left(x\left|x^{1}\right| / 2\right)\right| \leq\left|x^{1} / 2\right|^{2}|x|^{\gamma}$, so that $\left|\Delta v\left(x\left|x^{1}\right| / 2\right)\right| \leq C_{1}$ in $\mathcal{C} \cap\left(B_{3 / 4} \backslash B_{1 / 4}\right)$. We now restrict ourselves to the situation in which $x^{1} /\left|x^{1}\right|$ is uniformly bounded away from $\partial \mathcal{C}$. Since $v\left(x^{0}\left|x^{1}\right| / 2\right) \leq C_{2}\left|x^{1}\right|^{\alpha_{1}}$, we use the (interior) Harnack inequality on $\mathcal{C} \cap\left(B_{3 / 4} \backslash B_{1 / 4}\right)$ to conclude that

$$
v\left(x^{1}\right) \leq C_{3}\left(C_{2}\left|x^{1}\right|^{\alpha_{1}}+C_{1}\left|x^{1}\right|^{2+\gamma}\right) \leq C_{4}\left|x^{1}\right|^{\alpha_{1}},
$$

where in the second inequality we have used $2-\alpha_{1}+\gamma>0$, and $\left|x^{1}\right|<1$.

The constant $C_{3}$ does not remain bounded as $x^{1}$ approaches $\partial \mathcal{C}$. Furthermore, we also have that $f_{1}\left(x^{1} /\left|x^{1}\right|\right)$ goes to zero as $x^{1} /\left|x^{1}\right|$ approaches $\partial \mathcal{C} \cap S^{n-1}$. If $x^{1}$ is close to $\partial \mathcal{C}$, we employ the boundary Harnack principle (1.1) with right hand 
side as given in [5] which is applicable as long as $\partial \mathcal{C} \cap\left(B_{3 / 4} \backslash B_{1 / 4}\right) \in C^{1, \beta}$. We may then conclude that

$$
v\left(x^{1}\right) \leq C_{5} u\left(x^{1}\right)\left(\|v\|_{L^{\infty}}+\left|x_{1}\right|^{2+\gamma}\right) \leq C_{6} u\left(x^{1}\right)=C_{6}\left|x^{1}\right|^{\alpha_{1}} f_{1}\left(x^{1} /\left|x^{1}\right|\right),
$$

and this concludes the result.

As a corollary we are able to bound a supersolution by a subsolution as long as the right hand side is small enough and the subharmonic solution has a large enough height.

Corollary 2.3. Let $\mathcal{C}$ and $\gamma$ have the same assumptions as in Theorem 2.1. Let $u, v \geq 0$ with $u=v=0$ on $\partial \mathcal{C} \cap B_{1}$ and $u\left(x^{0}\right)=v\left(x^{0}\right)=1$. There exists $\varepsilon>0$ with the same dependence as $C$ in Theorem 2.1 such that if

$$
-C_{0}|x|^{\gamma} \leq \Delta u, \Delta v \leq \varepsilon|x|^{\gamma},
$$

then

$$
\frac{v(x)}{u(x)} \leq 4 C \text { for any } x \in \mathcal{C} \cap B_{1 / 2}
$$

where $C$ is the constant appearing in Theorem 2.1 .

Proof. Let $x^{0} \in \mathcal{C} \cap B_{3 / 4}$ and $w$ solve $\Delta w=\varepsilon|x|^{\gamma}$ in $\mathcal{C} \cap B_{3 / 4}$, with $w=0$ on $\partial\left(\mathcal{C} \cap B_{3 / 4}\right)$. We first observe that

$$
-\left(C_{0}+\varepsilon\right)|x|^{\gamma} \leq \Delta(u-w), \Delta(v-w) \leq 0 .
$$

For $\varepsilon$ small we have $c_{1} \varepsilon \leq\left|w\left(x^{0}\right)\right| \leq c_{2} \varepsilon$, with $c_{1}, c_{2}$ depending on $M$ and the $\operatorname{dist}\left(x^{0}, \partial\left(\mathcal{C} \cap B_{1}\right)\right)$. We also let $\tilde{u}$ be the harmonic replacement of $u$ in $B_{3 / 4} \cap \mathcal{C}$. Then $\tilde{u}\left(x^{0}\right)$ is bounded below by a constant with the same dependence as $C$ in Theorem 2.1. Then applying Theorem 2.1 to $\tilde{u}$ and $w$ we obtain that

$$
\frac{-w(x)}{\tilde{u}(x)} \leq C \frac{-w\left(x^{0}\right)}{\tilde{u}\left(x^{0}\right)} \leq C c_{2} \varepsilon \text { in } \mathcal{C} \cap B_{1 / 2} .
$$

Applying Theorem 2.1 to $v-w$ and $\tilde{u}$ we obtain

$$
(v-w)(x) \leq C \tilde{u}(x) .
$$

Since $\tilde{u}$ is the harmonic replacement of $u$ and $\Delta(u-w) \leq 0$ with $\tilde{u}=u-w$ on $\partial\left(\mathcal{C} \cap B_{3 / 4}\right)$, we have $\tilde{u} \leq u-w$, and hence by $(2.10)(v-w)(x) \leq C(u-w)(x)$ in $\mathcal{C} \cap B_{1 / 2}$. Now by (2.9) we have

$$
-w(x) \leq C c_{2} \varepsilon \tilde{u}(x) \leq C c_{2} \varepsilon(u-w)(x) .
$$

From these inequalities we conclude that

$$
(v-w)(x) \leq C(u-w)(x) \leq C u(x)-C w(x) \leq C u(x)-C^{2} c_{2} \varepsilon(u-w)(x) .
$$

We choose $\varepsilon<\left(2 C^{2} c_{2}\right)^{-1}$ so that

$$
v(x) \leq(v-w / 2)(x) \leq C u(x) \quad \text { in } \mathcal{C} \cap B_{1 / 2} .
$$


We also state here another result which will be needed later in the proof of Lemma 3.14:

Corollary 2.4. Let $\mathcal{C}$ and $\gamma$ have the same assumptions as in Theorem 2.1. Let $u$ solve

$$
\begin{aligned}
\Delta u & =0 \text { in } \mathcal{C} \cap B_{1} \\
u & =0 \text { on } \partial \mathcal{C} \cap B_{1} \\
u & \geq 0,
\end{aligned}
$$

and let $v$ solve (for some $\eta>0$ )

$$
\begin{aligned}
0 \leq \Delta v & \leq \eta \text { in } \mathcal{C} \cap B_{1} \\
v & =\text { u } \text { on } \partial\left(\mathcal{C} \cap B_{1}\right) .
\end{aligned}
$$

There exists $\eta_{0}>0$ with the same dependence as $C$ in Theorem 2.1 such that if $\eta \leq \eta_{0}$ then $v>0$ in $B_{r} \cap \mathcal{C}$ for $r$ small enough.

Proof. We let $x^{0}$ be as in Theorem 2.1. We note that $u\left(x^{0}\right)-v\left(x^{0}\right)$ is comparable to $\eta$, so we apply Theorem 2.1 to $(u-v) /\left(u\left(x^{0}\right)-v\left(x^{0}\right)\right)$ and $u / u\left(x^{0}\right)$ to obtain that

$$
\sup _{B_{1 / 2}} \frac{u-v}{u} \leq C \frac{u\left(x^{0}\right)-v\left(x^{0}\right)}{u\left(x^{0}\right)} \leq C_{1} \eta .
$$

Then for $C_{1} \eta<1 / 2$ we have that $0 \leq u \leq 2 w$ on $B_{1 / 2}$ and this concludes the proof.

We now show that the assumption that $2-\alpha_{1}+\gamma>0$ is essential. We first consider the easier case when $2-\alpha_{1}+\gamma<0$, and show that Theorem 2.1 cannot possibly hold. For clarity of exposition we restrict the analysis to the case when $\gamma=0$, so that the right hand side is constant.

Theorem 2.5. Let $u$ and $v$ be as in the statement of Theorem 2.1, and assume $\Delta v=-1$ with $2-\alpha_{1}<0$. Then, for any $C>0$, there exists $\rho>0$ such that

$$
C u<v \text { in } \mathcal{C} \cap B_{\rho} .
$$

Proof. We note that by the standard boundary Harnack inequality, it is enough to consider $u=r^{\alpha_{1}} f_{1}(\theta)$. We normalize $f_{1}$ so that $\sup f_{1}=1$. Fix $C>0$, and let $z \in B_{r} \cap \mathcal{C}$. Define $h(x):=|x-z|^{2} /(2 n)$. We note that $h$ is constant on $\partial B_{r}(z)$. If there exists $y \in \partial B_{r}(z)$ with $h(y) \leq C u(y)-v(y)$, then

$$
\frac{r^{2}}{2 n}=h(y) \leq C u(y)-v(y) \leq C r^{\alpha_{1}} .
$$

For small enough $r$, the above inequality cannot hold since $2<\alpha_{1}$. Then for small enough $r$, we have $h \geq C u-v$ on $\partial B_{r}(z)$, and hence also on $\partial\left(B_{r}(z) \cap \mathcal{C}\right)$. By the comparison principle $h \geq C u-v$ in $B_{r}(z) \cap \mathcal{C}$, and so $0=h(0) \geq C u(z)-v(z)$, so that $C u(z)-v(z) \leq 0$. Since $C$ was arbitrary, for any $\delta>0$, we may choose $r_{0}$ such that if $r<r_{0}$ and $x \in B_{r} \cap \mathcal{C}$, then $(C+\delta) u(x)-v(x) \leq 0$. Since $u>0$ in $B_{r} \cap \mathcal{C}$ it follows that $C u(x)-v(x)<0$ for any $x \in B_{r} \cap \mathcal{C}$. 
We can show that Theorem 2.1 is sharp by considering the critical case when $2-\alpha_{1}=0$. For when dimension $n=2$ this result was shown in [10].

Theorem 2.6. Let $\mathcal{C}$ be a cone in $\mathbb{R}^{n}$ with $\alpha_{1}=2$. Then the boundary Harnack principle with right hand side does not hold.

Proof. Let $v$ be as in the statement of the Theorem, and let $u=r^{\alpha_{1}} f_{1}(\theta)$. Suppose that there exists $C>0$ such that

$$
C^{-1} u(x) \leq v(x) \leq C u(x) \text { for all } x \in \mathcal{C} \cap B_{1 / 2} .
$$

We now use a Weiss-type monotonicity formula for superharmonic functions as in [8]. The function

$$
W(r, v):=\frac{1}{r^{n+2}} \int_{B_{r} \cap \mathcal{C}}\left(|\nabla v|^{2}-2 v\right)-\frac{2}{r^{n+3}} \int_{\partial B_{r} \cap \mathcal{C}} v^{2},
$$

is monotonically increasing in $r$ and is constant if and only if $v$ is homogeneous. We now consider the rescaled functions $v_{r}(x):=v(r x) / r^{2}$. From (2.11) and the fact that $u$ is homogeneous of degree 2 we have that, for any fixed $x \in \mathcal{C} \cap B_{1}$, there exists $C_{x}$ such that

$$
C_{x}^{-1} r^{2} \leq v_{r}(x) \leq C_{x} r^{2} \text { for any } 0<r<1 .
$$

From Lemma 2.2 we obtain that for a sequence $r_{k} \rightarrow 0, v_{r_{k}} \rightarrow v_{0}$ uniformly in $\mathcal{C} \cap B_{R}$ for any $R>0$. Furthermore, we will show that $v_{0}$ satisfies that

(i) $v_{0}$ is homogeneous of degree 2 ,

(ii) $v_{0} \geq 0$,

(iii) $v_{0}=0$ on $\partial \mathcal{C}$,

(iv) $\Delta v_{0}=-1$ in $\mathcal{C}$,

(v) $v$ is not identically zero.

Property $(i)$ follows from the Weiss-type monotonicity formula by checking that $W(\rho r, v)=W\left(\rho, v_{r}\right)$; since $W(r, v)$ is monotone in $r$, it follows that

$$
W\left(\rho, v_{0}\right)=\lim _{r_{k} \rightarrow 0} W\left(\rho, v_{r_{k}}\right)=\lim _{r_{k} \rightarrow 0} W\left(\rho r_{k}, v\right)=W(0+, v) \text { for any } \rho>0
$$

and since $W\left(\rho, v_{0}\right)$ is constant, then $v_{0}$ is homogeneous of degree 2, see [8]. Properties $(i i)-(i v)$ follow easily from the definition of $v_{r}$ and the uniform convergence. Finally, property $(v)$ follows from (2.12). Then $v_{0}=r^{2} g$ where the spherical piece $g$ satisfies $2 n g+\Delta_{\theta} g=-1$. We now utilize the Fredholm Alternative for existence for weak solutions, see Chapter 6 in [6]. Since $f_{1}$ (in (2.1)) is a nontrivial solution, the solution $g$ exists if and only if

$$
0=\langle-1, h\rangle=\int_{\mathcal{C} \cap \partial B_{1}}-h
$$


for all $h$ such that $2 n h+\Delta_{\theta} h=0$ (since the operator $2 n+\Delta_{\theta}$ is self-adjoint). We recall that $2 n f_{1}+\Delta_{\theta} f_{1}=0$. Then a necessary condition for $g$ to exist is that

$$
0=\left\langle-1, f_{1}\right\rangle=\int_{\mathcal{C} \cap \partial B_{1}} f_{1} .
$$

Since $f_{1}>0$ in $\mathcal{C} \cap \partial B_{1}$, the above equality cannot be true. Consequently, a solution $g$ cannot exist, and we have a contradiction.

\section{Boundary Harnack in Lipschitz Domains}

In this section we consider Lipschitz domains $D_{L, R}$ where

$$
D_{L, R}:=\left\{\left(x^{\prime}, x_{n}\right) \in B_{R}: x_{n}>g\left(x^{\prime}\right)\right\}
$$

and $g$ is Lipschitz with constant $L$, that is $\left|g\left(x^{\prime}\right)-g\left(y^{\prime}\right)\right| \leq L\left|x^{\prime}-y^{\prime}\right|$. We will assume $g(0)=0$, and will write $D_{L}$ when $R=1$ and $D_{L, \infty}$ if $R=\infty$. Also, we define $u \in \mathcal{S}\left(D_{L, R}\right)$ if

$$
\begin{aligned}
\Delta u(x) & =0 \text { in } D_{L, R}, \\
u(x) & =0 \text { on } D_{L, R}^{c} \cap B_{R},
\end{aligned}
$$

and for $\gamma \in \mathbb{R}$ we define $u \in \mathcal{S}\left(D_{L, R}, d^{\gamma}\right)$ if

$$
\begin{aligned}
|\Delta u(x)| & \leq\left(\operatorname{dist}\left(x, \partial D_{L, R} \cap B_{R}\right)\right)^{\gamma} & & \text { in } D_{L, R}, \\
u(x) & =0 & & \text { on } D_{L, R}^{c} \cap B_{R} .
\end{aligned}
$$

It will be necessary to use the solutions on right circular cones as barriers. Consequently, we define

$$
\mathcal{C}_{L}:=\left\{\left(x^{\prime}, x_{n}\right): x_{n}>L\left|x^{\prime}\right|\right\}
$$

If $u \in \mathcal{S}\left(\mathcal{C}_{L, \infty}\right)$ and $u \geq 0$, then as noted in Section 2, we have $u=r^{\alpha_{1}} f_{1}(\theta)$ (and unique up to multiplicative constant), and we will denote $r^{\alpha_{1}} f_{1}$ by $u_{L}$. We note that the definition of $\mathcal{C}_{(-L)}$ still makes sense when $-L<0$; although, the cone $\mathcal{C}_{(-L)}$ is not convex. In this situation we write $\mathcal{C}_{(-L)}$ and similarly $u_{(-L)}$ for the nonnegative solution with zero boundary data on $\mathcal{C}_{(-L)}$.

In order to prove a boundary Harnack principle with a right hand side for Lipschitz domains we will adapt the proof from Section 2 in the following manner:

- We will employ compactness methods and thus need a convergence result provided by Lemma 3.2.

- We will need to bound the behavior of a nonnegative harmonic function at the boundary from above and below which is given in Lemma 3.3.

- We will need a Liouville type result which is given in Lemma 3.6.

- We will then adapt the proof of Theorem 2.1 (again using compactness techniques) to obtain the proof of Theorem 3.10. 
Lemma 3.1. Let $L \leq M$ and $u \in \mathcal{S}\left(D_{L}\right)$ with $u \geq 0$. Let $x \in \partial D_{L} \cap B_{1 / 2}$ and let $y \in D_{L}$ with $\operatorname{dist}\left(y, \partial D_{L}\right)>\delta$. Then there exists a constant $C=C(n, M, \delta)$ such that

$$
\begin{aligned}
& \sup _{B_{r}(x)} u \leq C u(y) \quad \text { for all } r \leq 1 / 4, \\
& \sup _{B_{1 / 4}} u \geq C^{-1} u(y) .
\end{aligned}
$$

We give later a proof of a more general version of this lemma; see Lemma 4.4 in Section 4.

Lemma 3.2. Let $D_{L_{k}, R_{k}}$ be a sequence of domains with $L_{k} \leq M, R_{k} \geq 1$, and $0 \in \partial D_{L_{k}}$. Let $u_{k} \in \mathcal{S}\left(D_{L_{k}, R_{k}}, d^{\gamma}\right)$, and let $\alpha$ be the degree of homogeneity for $u_{M}$, and assume $2-\alpha+\gamma>0$. We further assume either

(1) $u_{k} \geq 0$ and $\sup u_{k} \leq 1$, or

$$
B_{1 / 2}
$$

(2) $\sup u_{k} \leq C r^{\beta}$ for $r \leq 1$ and some constants $C, \beta>0$.

$$
B_{r}
$$

Then there exists a subsequence with a limiting domain $D_{L_{0}, R_{0}}$ and a limiting function $u_{0} \in \mathcal{S}\left(D_{L_{0}, R_{0}}, d^{\gamma}\right)$ such that

$$
\sup _{B_{r}}\left|u_{k}-u_{0}\right| \rightarrow 0 \quad \text { as } k \rightarrow \infty
$$

for all $r<R_{0}$.

We give later a proof of the more general Lemma 3.2 in Section 4 that implies Lemma 3.2.

Lemma 3.3. Let $u \in \mathcal{S}\left(D_{L}\right)$ with $u \geq 0$. Let $L<M$ and let $\alpha_{1}$ be the degree of homogeneity for $u_{M}$ and $\beta$ the degree of homogeneity for $u_{(-M)}$. There exist constants $c_{1}, c_{2}$ depending only on $n, M$, and $M-L$ such that for any $x \in \partial D_{L} \cap$ $B_{1 / 2}$,

$$
\begin{aligned}
& \text { (1) } \sup _{B_{r}(x)} u \geq c_{1} u\left(e_{n} / 2\right) r^{\alpha_{1}} \text {, } \\
& \text { (2) } \sup _{B_{r}(x)} u \leq c_{2} u\left(e_{n} / 2\right) r^{\beta}
\end{aligned}
$$

for any $r \leq 1 / 4$.

Proof. Let $x \in \partial D_{L} \cap B_{1 / 2}$. From Lemma 3.1 we have $u(y) \geq C_{1} u\left(e_{n} / 2\right)$ for any $y \in \partial B_{1 / 4}(x) \cap\left(\mathcal{C}_{M}+x\right)$. We now consider the translated function $u_{M}(y-x)$ which is unique up to multiplicative constant and homogeneous of degree $\alpha_{1}$. By multiplying $u_{M}(y-x)$ by a positive constant, we may assume that $\sup _{B_{1 / 4}(x)} u_{M}(y-$ $x)=C_{1} u\left(e_{n} / 2\right)$. Then $u_{M}(y-x) \leq u(y)$ on $\partial B_{1 / 4}(x) \cap\left(\mathcal{C}_{M}+x\right)$. Since $u_{M}(y-$ 
$x)=0$ on $\partial\left(\mathcal{C}_{M}+x\right)$, we may invoke the comparison principle to conclude that $u_{M}(y-x) \leq u(y)$ on $B_{1 / 4}(x) \cap\left(\mathcal{C}_{M}+x\right)$. Then

$$
\sup _{B_{r}(x)} u(y) \geq \sup _{B_{r}(x)} u_{M}(y-x)=C_{1} u\left(e_{n} / 2\right) r^{\alpha_{1}},
$$

which proves (1). In a similar manner we obtain property (2) by bounding $u$ from above by $u_{(-M)}$.

Corollary 3.4. (To Lemma 3.3) Let $u \in \mathcal{S}\left(D_{L}\right)$ with $L<M$, and let $\beta$ be the degree of homogeneity for $u_{(-M)}$. There exists a constant $C$ depending only on dimension $n, M$, and $M-L$ such that for any $x \in \partial D_{L} \cap B_{1 / 2}$,

$$
\sup _{B_{r}(x)}|u| \leq C\left(\sup _{B_{1}}|u|\right) r^{\beta}
$$

for any $r \leq 1 / 4$.

Proof. We consider the solution

$$
\begin{aligned}
\Delta v & =0 & & \text { in } D_{L}, \\
v & =0 & & \text { on } D_{L}^{c} \cap B_{1}, \\
v & =\sup _{B_{1}} u & & \text { on } \partial B_{1} \cap D_{L} .
\end{aligned}
$$

From Lemma 3.3 we have that

$$
v \leq C v(0,1 / 2) r^{\beta} \leq C\left(\sup _{B_{1}} v\right) r^{\beta} .
$$

From the comparison principle we have that $u \leq v$ on $B_{1}$. By considering $-v$ we obtain a similar bound from below to conclude the proof.

Remark 3.5. A rescaling and translation to the origin of Corollary 3.4 shows that if $L<M$ and $\beta$ is the degree of homogeneity for $u_{(-M)}$, then if $u \in \mathcal{S}\left(D_{L}, R\right)$ with $R>2$, then

$$
\left.\sup _{B_{1}}|u|\right) R^{\beta} \leq c_{2} \sup _{B_{R}}|u| .
$$

Lemma 3.6. Let $u, v \in \mathcal{S}\left(D_{L, \infty}\right)$ with $u, v \geq 0$, then $u=c v$ for some constant $c \geq 0$.

Proof. Consider $w=u+v$. Then $w \geq u$. Let $c \geq 1$ be the largest constant such that $c u \leq w$. Then there exists a sequence $\left\{x_{k}\right\} \in D_{L, \infty}$ such that

$$
\lim _{k \rightarrow \infty} \frac{c u\left(x_{k}\right)}{w\left(x_{k}\right)}=1 .
$$

We now invoke the standard boundary Harnack principle for Lipschitz domains on the nonnegative harmonic functions $w-c u$ and $w$. There exists $C_{1}>0$ such that

$$
\sup _{B_{r_{k}}} \frac{w-c u}{w} \leq C_{1} \inf _{B_{r_{k}}} \frac{w-c u}{w} \leq C_{1}\left(1-\frac{c u\left(x_{k}\right)}{w\left(x_{k}\right)}\right),
$$

with $r_{k}:=\max \left\{2\left|x_{k}\right|, k\right\}$. From (3.1) the right hand side of (3.2) goes to zero. Then $w \equiv c u$, and the result follows. 
Lemma 3.7. Let $u, v \in \mathcal{S}\left(D_{L, \infty}\right)$ with $u \geq 0$. If there exist constants $C, R>0$ such that

$$
\sup _{B_{r}}|v| \leq C \sup _{B_{r}} u \quad \text { for } r \geq R,
$$

then $v=$ cu for some $c \in \mathbb{R}$.

Remark 3.8. The significance of Lemma 3.7 is that we do not require $v \geq 0$.

Proof. Let $v_{r}$ satisfy

$$
\begin{aligned}
\Delta v_{r} & =0 \quad \text { in } B_{r} \cap D_{L, \infty} \\
v_{r} & =v^{+} \text {on } \partial\left(B_{r} \cap D_{L, \infty}\right) .
\end{aligned}
$$

By Lemma 3.3 we have that

$$
\sup _{B_{2 r}}|v| \leq C \sup _{B_{r}}|v| \text { for } r \geq 1
$$

and some constant $C$ independent of $r$. Then

$$
v_{r}(r / 2,0, \ldots, 0) \leq C \sup _{B_{r}}|v| \leq C \sup _{B_{r / 2}}|v| \leq C \sup _{B_{r / 2}} u \leq C u(r / 2,0, \ldots, 0),
$$

with the last inequality following form Lemma 3.1. By the standard boundary Harnack principle,

$$
\sup _{B_{r / 2}} \frac{v^{+}}{u} \leq \sup _{B_{r / 2}} \frac{v_{r}}{u} \leq C \frac{v(r / 2,0, \ldots, 0)}{u(r / 2,0, \ldots, 0)} \leq C_{1} .
$$

Since the constants are independent of $r \geq 1$, we conclude that $v^{+} \leq C_{1} u$ in $D_{L, \infty}$. The same argument holds for $v^{-}$so that $|v| \leq C_{1} u$ in $D_{L, \infty}$.

Let $w=C_{1} u-v \geq 0$. Then from Lemma 3.6 we have that $w=c u$ for some constant $c$, so that $v=\left(C_{1}-c\right) u$.

We will need an improvement over the previous lemma.

Lemma 3.9. Let $u, v \in \mathcal{S}\left(D_{L, \infty}\right)$ with $u \geq 0$. If there exist constants $C, R>0$ such that

$$
\sup _{B_{r}}|v| \leq C(\ln (r+1)) \sup _{B_{r}} u \quad \text { for } r \geq 1,
$$

then $v=$ cu for some $c \in \mathbb{R}$.

In Section 4 we give a proof of a more general result in Theorem 4.2. 
Theorem 3.10. Let $0 \in \partial D_{L, 2}$ with $L<M$, and fix $x^{0} \in D_{L}$. Assume further that $B_{1} \cap\left\{x_{n}>1 / 4\right\} \subseteq D_{L, 2}$. Let $\alpha_{1}$ be the degree of homogeneity for $u_{M}$, and assume that $2-\alpha_{1}+\gamma>0$. Let $u, v \geq 0$ with $u\left(x^{0}\right)=v\left(x^{0}\right)=1$. If

$$
-C_{0}\left(\operatorname{dist}\left(x, \partial D_{L, R} \cap B_{R}\right)\right)^{\gamma} \leq \Delta u, \Delta v \leq \varepsilon\left(\operatorname{dist}\left(x, \partial D_{L, R} \cap B_{R}\right)\right)^{\gamma},
$$

then there exists constants $C, \varepsilon>0$ (depending only on dimension $n, C_{0}$, Lipschitz constant $M, M-L$, and $\left.\operatorname{dist}\left(x^{0}, \partial D_{L, 2}\right)\right)$ such that

$$
C^{-1} v(x) \leq u(x) \leq C v(x)
$$

for all $x \in B_{1 / 2}$.

Proof. For simplicity we assume $\Delta u=0$ and $\Delta v \leq 0$ in $D_{L, 2}$ and show that $v \leq C u$ in $B_{1 / 2}$. Once this has been shown, the situation when $u$ and $v$ satisfy (3.3) is proven exactly as in Corollary 2.3. We also initially prove the theorem for a fixed $x^{0} \in \mathcal{C}_{M} \cap \partial B_{1 / 2}$.

Suppose by way of contradiction that the theorem is not true. Then there exists $u_{k}, v_{k}$ satisfying $\Delta u_{k}=0$ and

$$
\Delta v_{k} \geq-\left(\operatorname{dist}\left(x, \partial D_{L, R} \cap B_{R}\right)\right)^{\gamma},
$$

with $v_{k}\left(x^{0}\right)=u_{k}\left(x^{0}\right)=1$ and points $x_{k} \in D_{L_{k}, 2} \cap B_{1 / 2}$ such that

$$
k u_{k}\left(x_{k}\right)<v_{k}\left(x_{k}\right) .
$$

Harnack chains work on Lipschitz domains; therefore, from the interior Harnack inequality, by multiplying $v_{k}$ and $u_{k}$ by constants (uniformly bounded above and below), we may assume that

$$
v_{k}\left(x_{k}^{\prime}, e_{n}\right)=u_{k}\left(x_{k}^{\prime}, e_{n}\right)=1 .
$$

Because of the interior Harnack inequality, and by the fact that $\varepsilon_{k} \rightarrow 0$, in order for (3.5) to occur, it is necessary that $\operatorname{dist}\left(x_{k}, \partial D_{L_{k}}\right) \rightarrow 0$. We let $y_{k}=\left(x_{k}^{\prime}, e_{n}\right)$, and similar to the proof of Theorem 2.1 we define

$$
w_{r, k}(x):=\frac{v_{k}(r x)-\frac{v_{k}\left(r y_{k}\right)}{u_{k}\left(r y_{k}\right)} u_{k}(r x)}{\sup _{B_{1} \cap D_{L_{k}}}\left|v_{k}(r x)-\frac{v_{k}\left(r y_{k}\right)}{u_{k}\left(r y_{k}\right)} u_{k}(r x)\right|},
$$

and

$$
a_{k, m}:=\sup _{B_{1} \cap D_{L_{k}}}\left|v_{k}\left(2^{-m} x\right)-\frac{v_{k}\left(2^{-m} y_{k}\right)}{u_{k}\left(2^{-m} y_{k}\right)} u_{k}\left(2^{-m} x\right)\right|\left(\sup _{B_{2}-m} u_{k}\right)^{-1}
$$

For fixed $m$, it follows by the standard boundary Harnack Principle that

$$
b_{m}:=\sup _{k} a_{k, m}<\infty .
$$


Just as in the proof of Theorem 2.1, by the assumption (3.5) we necessarily obtain that

$$
\sum b_{m}=\infty
$$

From Lemma A. 1 there exists a subsequence $b_{m_{l}}$ such that for any fixed $j$ we have

$$
\limsup _{m_{l} \rightarrow \infty} \frac{\sum_{i=1}^{j} b_{m_{l}-i}}{b_{m_{l}}} \leq j \text {. }
$$

For any $N>0$ with $N \in \mathbb{N}$, there exists $\tilde{N} \in \mathbb{N}$, such that if $m_{l} \geq \tilde{N}$, then there is a $k=k\left(m_{l}\right)$ such that $a_{k, m_{l}}$ satisfies

$$
\frac{\sum_{i=1}^{j} a_{k, m_{l}-i}}{a_{m_{l}}} \leq C j \text { for } j \leq N .
$$

If we let $r_{k}=2^{-m_{l}}$, then for those chosen $k$ we have that

$$
\sup _{B_{2 j}}\left|w_{r_{k}, k}(x)\right|=\frac{\sup _{B_{2 j}}\left|v_{k}\left(r_{k} x\right)-\frac{v_{k}\left(r_{k} y_{k}\right)}{u_{k}\left(r_{k} y_{k}\right)} u_{k}\left(r_{k} x\right)\right|}{\sup _{B_{1} \cap D_{L_{k}}}\left|v_{k}(r x)-\frac{v_{k}\left(r y_{k}\right)}{u_{k}\left(r y_{k}\right)} u_{k}(r x)\right|},
$$

and

$$
\begin{aligned}
& \left|\frac{v_{k}\left(2^{j-i} r_{k} y_{k}\right)}{u_{k}\left(2^{j-i} r_{k} y_{k}\right)} u_{k}\left(2^{j-i-1} r_{k} y_{k}\right)-\frac{v_{k}\left(2^{j-i-1} r_{k} y_{k}\right)}{u_{k}\left(2^{j-i-1} r_{k} y_{k}\right)} u_{k}\left(2^{j-i-1} r_{k} y_{k}\right)\right| \\
& \quad=\left|\frac{v_{k}\left(2^{j-i} r_{k} y_{k}\right)}{u_{k}\left(2^{j-i} r_{k} y_{k}\right)} u_{k}\left(r_{k} 2^{j-i-1} y_{k}\right)-v_{k}\left(2^{j-i-1} r_{k} y_{k}\right)\right| \\
& \leq a_{k, m_{l}-(j-i)} \sup _{B_{2 j-i} r_{k}} u_{k} .
\end{aligned}
$$

Also we have that

$$
\begin{aligned}
\sup _{B_{2 j}} & \left|\frac{v_{k}\left(2^{j-i} r_{k} y_{k}\right)}{u_{k}\left(2^{j-i} r_{k} y_{k}\right)} u_{k}\left(r_{k} x\right)-\frac{v_{k}\left(2^{j-i-1} r_{k} y_{k}\right)}{u_{k}\left(2^{j-i-1} r_{k} y_{k}\right)} u_{k}\left(r_{k} x\right)\right| \\
= & \sup _{B_{2^{j}}} \frac{u_{k}\left(r_{k} x\right)}{u_{k}\left(2^{j-i-1} r_{k} y_{k}\right)} \\
& \times\left|\frac{v_{k}\left(2^{j-i} r_{k} y_{k}\right)}{u_{k}\left(2^{j-i} r_{k} y_{k}\right)} u_{k}\left(2^{j-i-1} r_{k} y_{k}\right)-\frac{v_{k}\left(2^{j-i-1} r_{k} y_{k}\right)}{u_{k}\left(2^{j-i-1} r_{k} y_{k}\right)} u_{k}\left(2^{j-i-1} r_{k} y_{k}\right)\right| \\
\leq & \sup _{B_{2 j}} \frac{u_{k}\left(2^{j} r_{k} x\right)}{u_{k}\left(2^{j-i-1} r_{k} y_{k}\right)} a_{m_{l}-(j-i)} \sup _{B_{2}-i r_{k}} u_{k} \\
\leq & C a_{m_{l}-(j-i)} \frac{u_{k}\left(2^{j} r_{k} y_{k}\right)}{u_{k}\left(2^{j-i-1} r_{k} y_{k}\right)} u_{k}\left(2^{j-i} r_{k} y_{k}\right) \\
\leq & C a_{m_{l}-(j-i)} u_{k}\left(2^{j} r_{k} y_{k}\right),
\end{aligned}
$$


with the last inequality following from the bounds in Lemma 3.3. Then, combining estimates (3.7) and (3.8), we obtain

$$
\sup _{B_{2 j}}\left|w_{r_{k}, k}(x)\right| \leq C j \frac{\sup _{B_{2 j}} u_{k}\left(r_{k} x\right)}{\sup _{B_{1}} u_{k}\left(r_{k} x\right)} .
$$

We now use Lemma 3.2 as $r_{k} \rightarrow 0$ to obtain limiting functions and domains. By choosing a subsequence we first consider the limit function

$$
u=\lim _{r_{k} \rightarrow 0} \frac{u_{k}\left(r_{k} x\right)}{\sup _{B_{r_{k}}} u_{k}} .
$$

We also obtain a limiting global Lipschitz domain $D$ on which $u$ is the unique (up to multiplicative constant) nonnegative harmonic function that vanishes on the boundary. A further subsequence guarantees $y_{k} \rightarrow y_{0} \in D$. As in the proof of Theorem 2.1, as $r_{k} \rightarrow 0$ we have that $\left|\Delta w_{r_{k}, k}\right| \rightarrow 0$. Then by picking a further subsequence, as $r_{k} \rightarrow 0$ we obtain a limiting global Lipschitz domain function $w$ with the following properties:

(i) $\Delta w=0$ in $D$ and $w=0$ on $D^{c}$,

(ii) $\sup |w|=1$, $B_{1}$

(iii) $w\left(y^{0}\right)=0$,

(iv) $w(x) \leq C u(x) \ln (|x|+1)$ for $|x| \geq 1$ from (3.9).

From property (iv) Lemma 3.9 guarantees $w(x)=c u(x)$, but then both properties (ii) and (iii) cannot hold since $u\left(y^{0}\right)>0$.

An interior Harnack inequality with a Harnack chain will also give the result for $x^{0} \in B_{1 / 2} \cap D_{L}$ and the constant $C$ depending on $\operatorname{dist}\left(x^{0}, \partial D_{L}\right)$.

Remark 3.11. If one does not assume that $B_{1} \cap\left\{x_{n}>1 / 4\right\} \subseteq D_{L}$, then one may modify the proof at the expense that (3.4) holds for $x \in B_{r}$ with $r \leq$ $\min \left\{(2 L)^{-1}, 1 / 2\right\}$.

To show that the quotient is not only bounded but also Hölder continuous up to the boundary, we start with the following Lemma:

Lemma 3.12. Let $D$ be a Lipschitz domain with small Lipschitz constant $c \leq c(n)$ and the origin $0 \in \partial D$. There exists $\varepsilon, \beta>0$ such that if

$$
\begin{aligned}
& |\Delta u|,|\Delta v| \leq \varepsilon \quad \text { in } D \cap B_{1}, \\
& u=v=0 \quad \text { on } \partial D \cap B_{1}, \\
& u, v \geq 0,
\end{aligned}
$$

and $u\left(x^{0}\right)=v\left(x^{0}\right)=1$ for some $x^{0} \in D \cap B_{1}$, thus

$$
\left\|\frac{v}{u}\right\|_{C^{0, \beta}\left(B_{1 / 2} \cap D_{L}\right)} \leq C,
$$

where the constants $C, \varepsilon, \beta$ depend on $n$ and $\operatorname{dist}\left(x^{0}, \partial\left(D \cap B_{1 / 2}\right)\right)$. 
Proof. We let $C>2$ be the largest of $C$ from Theorem 3.10 and $1 / c_{1}$ from Lemma 3.3. From Lemma 3.3 we have that

$$
\sup _{B_{r}} u, v \geq \frac{r^{\alpha_{1}}}{C} \text { in } B_{1 / 2}
$$

As the Lipschitz constant $M$ of the domain becomes smaller, the growth exponent $\alpha_{1} \geq 1$ becomes closer to 1 . By choosing $M$ small enough, we may assume $\alpha_{1}$ is close to 1 . Then

$$
\frac{2^{-2 k}}{\left(1-(2 C)^{-1}\right)^{k}\left(C-C^{-1}\right) \sup _{B_{2}-k} u} \leq \frac{C 2^{-2 k}}{\left(1-(2 C)^{-1}\right)^{k}\left(C-C^{-1}\right) 2^{-\alpha_{1} k}} \leq 2 .
$$

We also choose $\varepsilon$ small enough so that $\varepsilon 2 C<\tilde{\varepsilon}$ where $\tilde{\varepsilon}$ is the $\varepsilon$ given in Theorem 3.10. We now follow the proof of Lemma 11.8 in [4]. We will show that there exists two sequences $a_{k}, b_{k}$ with $C^{-1} \leq a_{k} \leq b_{k} \leq C$ such that on $D \cap B_{2^{-k}}$

$$
a_{k} u \leq v \leq b_{k} u \quad \text { and } \quad\left(b_{k}-a_{k}\right)=\left(1-\frac{1}{2 C}\right)\left(b_{k-1}-a_{k-1}\right) .
$$

We let $a_{1}=C^{-1}$ and $b_{1}=C$, and this base case is true by Theorem 3.10. We suppose that $a_{k}$ and $b_{k}$ have been chosen inductively. We define $\bar{u}:=u\left(2^{-k} x\right)$ and $\bar{v}:=v\left(2^{-k} x\right)$ and also define the positive functions

$$
\begin{aligned}
w_{1}(x) & :=\frac{\bar{v}-a_{k} \bar{u}}{b_{k}-a_{k}}, \\
w_{2}(x) & :=\frac{b_{k} \bar{u}-\bar{v}}{b_{k}-a_{k}} .
\end{aligned}
$$

Now either $w_{1}\left(e_{n} / 2\right)>\bar{u}\left(e_{n} / 2\right) / 2$ or $w_{2}\left(e_{n} / 2\right)>\bar{u}\left(e_{n} / 2\right) / 2$ since $w_{1}+w_{2}=\bar{u}$. We suppose that $w_{1}\left(e_{n} / 2\right)>\bar{u}\left(e_{n} / 2\right) / 2$. The key difference in this proof with a right hand side is to note that, by (3.11), and by choosing $\varepsilon$ small, we have that

$$
\left|\frac{\Delta w_{1}}{\bar{u}\left(e_{n} / 2\right)}\right| \leq \varepsilon \quad \text { in } B_{1},
$$

so that we also have that

$$
\left|\frac{\Delta w_{1}}{w_{1}\left(e_{n} / 2\right)}\right| \leq 2 \varepsilon \quad \text { in } B_{1}
$$

We now apply Theorem 3.10 to $w_{1} / w_{1}\left(e_{n} / 2\right)$ and $\bar{u} / \bar{u}\left(e_{n} / 2\right)$ to conclude that

$$
\frac{\frac{w_{1}}{w_{1}\left(e_{n} / 2\right)}}{\frac{\bar{u}}{\bar{u}\left(e_{n} / 2\right)}} \geq \frac{1}{C} \text { on } B_{1 / 2} \cap D \text {. }
$$

Thus we also have that

$$
\frac{1}{C} \leq \frac{\frac{2 w_{1}}{\bar{u}\left(e_{n} / 2\right)}}{\frac{\bar{u}}{\bar{u}\left(e_{n} / 2\right)}}=\frac{2 w_{1}}{\bar{u}} \text { on } B_{1 / 2} \cap D .
$$


Renormalizing back to $B_{2-(k+1)}$, we have that

$$
\frac{v-a_{k} u}{\left(b_{k}-a_{k}\right) u} \geq \frac{1}{2 C},
$$

that is, in $B_{2^{-(k+1)}}$,

$$
\left[a_{k}+\frac{1}{2 C}\left(b_{k}-a_{k}\right)\right] u \leq v \leq b_{k} u,
$$

so $b_{k+1}=b_{k}$ and $a_{k+1}+\frac{1}{2 C}\left(b_{k}-a_{k}\right)$.

This proves the Hölder estimate of $u / v$ up to the boundary along rays in the $e_{n}$ direction. The quotient $u / v$ is Hölder continuous in the interior from the usual interior estimates for $u$ and $v$. This concludes the proof.

Lemma 3.13. Let $D$ and $u$, $v$ have the same assumptions as in Lemma 3.12, except we now allow that

$$
-C_{0} \leq \Delta u, \Delta v \leq \varepsilon \text { in } D \cap B_{1} .
$$

Then

$$
\left\|\frac{v}{u}\right\|_{C^{0, \beta}\left(B_{1 / 2} \cap D_{L}\right)} \leq C
$$

where $C, \varepsilon, \beta$ have the same dependence as in Lemma 3.12.

Proof. The main point to consider is that now $-M \leq \Delta u, \Delta v \leq \varepsilon$. Since $u, v \geq 0$, by Theorem 3.10 both $u$ and $v$ are comparable to harmonic functions and so are bounded away from zero in the interior. From interior Hölder estimates the quotient $u / v$ is Hölder continuous in the interior. If $y$ is close to the boundary, then by rewriting it as $y=y^{\prime}+y_{n} e_{n}$, we rescale and translate as follows:

$$
\bar{u}:=\frac{u\left(y^{\prime}+y_{n} x\right)}{\sup _{B_{y_{n}}\left(y^{\prime}, 0\right)} u}, \quad \bar{v}:=\frac{v\left(y^{\prime}+y_{n} x\right)}{\sup _{B_{y_{n}}\left(y^{\prime}, 0\right)} m} .
$$

From Lemma 3.3 we have that

$$
\sup _{B_{y_{n}}\left(y^{\prime}, 0\right)} u \geq c y_{n}^{\alpha_{1}}
$$

Since $\alpha_{1}<2$, we have, for $y_{n}$ small enough, that $|\Delta \bar{u}|,|\Delta \bar{v}| \leq \varepsilon$. By applying Lemma 3.12 we have that $\bar{u} / \bar{v}$ is Hölder continuous up to the boundary. Rescaling backward we obtain that $u / v$ is Hölder continuous up to the boundary.

For the application in Section 1.4.2 we actually need to consider the quotient $v / u$ where $v$ is not required to have a sign and $|\Delta v| \leq M$. This is given in Theorem 1.1. We will need a decay rate for $v$ near points $x \in \partial D \cap \partial\{v>0\} \cap \partial\{v<0\}$. 
Lemma 3.14. Let $\varepsilon$ be as in Theorem 1.1 and $D_{L}$ as in Theorem 1.1. Let $u$ satisfy

$$
\begin{aligned}
-C_{0} & \leq \Delta u \leq \varepsilon \text { in } D_{L}, \\
u & =0 \text { on } \partial D_{L}, \\
u & \geq 0 \text { in } D_{L}, \\
u\left(e_{n} / 2\right) & =1 .
\end{aligned}
$$

Let $v$ satisfy

$$
\begin{aligned}
|\Delta v| & \leq C_{0} \text { in } D_{L}, \\
v & =0 \text { on } \partial D_{L}, \\
|v| & \leq 1 \text { in } D_{L},
\end{aligned}
$$

with $0 \in \partial D \cap \partial\{v>0\} \cap \partial\{v<0\}$. Then there exists $C, \delta_{0}>0$ depending on $n, C_{0}$ such that

$$
\sup _{B_{r}}|v| \leq C \sup _{B_{r}} r^{\delta_{0}} u \text { for all } r \leq 1 / 4
$$

Proof. Suppose by way of contradiction that the Lemma is not true. Then there exists sequences $u_{k}, v_{k}$ satisfying the assumptions of the Lemma and points $x_{k} \in$ $D_{L_{k}} \cap B_{1 / 4}$ and $\delta_{k} \rightarrow 0$ such that

$$
\left|v_{k}\left(x_{k}\right)\right| \geq k\left|x_{k}\right|^{\delta_{k}} u_{k}\left(x_{k}\right) .
$$

By Theorem 3.10 we may bound the quotient $v_{k}^{ \pm} / u$ uniformly on $B_{1 / 4}$, so it necessarily follows that $\left|x_{k}\right| \rightarrow 0$. We let $r_{k}:=\left|x_{k}\right|$. Furthermore, we may choose $x_{k}$ such that

$$
\left|v_{k}(x)\right| \leq 2 k|x|^{\delta_{k}} u_{k}(x) \text { in } B_{1 / 2} \backslash B_{r_{k}},
$$

otherwise we simply choose new points in $B_{1 / 4} \backslash B_{r_{k}}$ satisfying (3.12). We rescale by letting

$$
\bar{u}_{k}(x):=\frac{u_{k}\left(r_{k} x\right)}{\sup _{B_{r_{k}}} u} \quad \bar{v}_{k}(x):=\frac{v_{k}\left(r_{k} x\right)}{\sup _{B_{r_{k}}}|v|} .
$$

Then $\bar{u}_{k} \rightarrow u_{0}$ uniformly on compact sets with $\sup u_{0}=1$. By (3.12) and (3.13) we have that

$$
B_{1}
$$

$$
\begin{aligned}
\bar{v}_{k}(x) & =\frac{v_{k}\left(r_{k} x\right)}{\sup _{B_{r_{k}}}|v|} \leq \frac{2 k r_{k}^{\delta_{k}}|x|^{\delta_{k}} u_{k}(x)}{\sup _{B_{r_{k}}}|v|} \\
& \leq \frac{2 k r_{k}^{\delta_{k}}|x|^{\delta_{k}} u_{k}(x)}{k r_{k}^{\delta_{k}} \sup _{B_{r_{k}}} u}=2|x|^{\delta_{k}} \bar{u}_{k} .
\end{aligned}
$$

Then $\bar{v}_{k} \rightarrow v_{0}$ uniformly on compact sets. Since $D_{L_{k}}$ have small Lipschitz constant we also have that in the limit $\Delta u_{0}=\Delta v_{0}=0$ in the limiting Lipschitz domain 
$D_{0}$. From Lemma 3.7 or Lemma 3.9 we have that $u_{0}=v_{0}$. Hence $\left(\bar{v}_{k}\right)^{-} \rightarrow 0$ uniformly. We choose $k$ large enough so that $\left|\Delta \bar{u}_{k}\right|,\left|\Delta \bar{u}_{k}\right| \leq \eta$. We let $w_{k}^{1}$ satisfy

$$
\begin{aligned}
\Delta w_{k}^{1} & =-\eta \text { in } B_{1} \cap\left\{\bar{u}_{k}>0\right\}, \\
w_{k}^{1} & =\bar{v}_{k}^{-} \text {on } \partial\left(B_{1} \cap\left\{\bar{u}_{k}>0\right\}\right),
\end{aligned}
$$

$w_{k}^{2}$ satisfy

$$
\begin{aligned}
\Delta w_{k}^{2} & =0 \text { in } B_{1} \cap\left\{\bar{u}_{k}>0\right\}, \\
w_{k}^{2} & =\bar{v}_{k}^{-} \text {on } \partial\left(B_{1} \cap\left\{\bar{u}_{k}>0\right\}\right),
\end{aligned}
$$

and $w_{k}^{3}$ satisfy

$$
\begin{aligned}
\Delta w_{k}^{3} & =\eta \text { in } B_{1} \cap\left\{\bar{u}_{k}>0\right\}, \\
w_{k}^{3} & =\bar{v}_{k}^{+} \text {on } \partial\left(B_{1} \cap\left\{\bar{u}_{k}>0\right\}\right) .
\end{aligned}
$$

For $\eta$ chosen small enough, we have that $w_{k}^{3}>0$ in $\left\{\bar{u}_{k}>0\right\}$. This is a consequence of the analogue of Corollary 2.4 for Theorem 3.10. Now $w_{k}^{1}\left(e_{n} / 2\right)$ is comparable to $\eta$ with a constant depending only on $n$ and the Lipschitz constant of the domain. We have chosen $w_{k}^{1}, w_{k}^{2}$ so that by normalizing by a constant we may apply Theorem 3.10 and conclude that

$$
\frac{w_{k}^{1}}{w_{k}^{3}} \leq C w_{k}^{1}\left(e_{n} / 2\right) \quad \text { and } \quad \frac{w_{k}^{2}}{w_{k}^{3}} \leq C w_{k}^{2}\left(e_{n} / 2\right) \quad \text { in } B_{1 / 4} .
$$

Then, for $k$ large enough, we have that $w_{k}^{3}-w_{k}^{1}-w_{k}^{2}>0$ in $B_{1 / 4}$, but we also have that $v_{k} \geq w_{k}^{3}-w_{k}^{1}-w_{k}^{2}$. Hence $0 \notin \partial\left\{v_{k}<0\right\}$, and this is a contradiction.

Now we give the proof of Theorem 1.1.

Proof of Theorem 1.1. From Lemma 3.14 we have a Hölder growth rate whenever $x^{0} \in \partial D \cap \partial\{v>0\} \cap \partial\{v<0\}$. We now assume that $x^{0} \in \partial D$ but $x^{0} \notin \partial\{v<0\}$, and without loss of generality we set $x^{0}=0$. Then $v>0$ in $D \cap B_{r}$ for $r$ small. Suppose that there exists infinitely many $r_{k} \rightarrow 0$ such that

$$
\frac{v\left(r_{k} e_{n}\right)}{u\left(r_{k} e_{n}\right)}>r_{k}^{1 / 2} \text {. }
$$

By Theorem 3.10 the function $u$ is comparable to a harmonic function, and so we may apply Lemma 3.3 to $u$. Furthermore, since the Lipschitz constant for $D$ is small, the constant $\alpha_{1}$ in Lemma 3.3 is close to 1; therefore, we may conclude that

$$
\sup _{B_{r}} u \geq c r^{3 / 4},
$$

in a neighborhood of the origin. When rescaling by $v_{r}=u(r x)$ we have $\Delta v_{r}=$ $r^{2} \Delta v(r x)$, so that

$$
\frac{\Delta v_{r_{k}}(x)}{v_{r_{k}}\left(e_{n}\right)}=\frac{r_{k}^{2} \Delta v\left(r_{k} x\right)}{v_{r_{k}}\left(e_{n}\right)} \leq \frac{r_{k}^{3 / 2} \Delta v\left(r_{k} x\right)}{u\left(r_{k} e_{n}\right)} \leq \frac{C_{0}}{c} r_{k}^{3 / 4} .
$$


Therefore, for $r_{k}$ small enough we may apply Theorem 1.1 to $v_{r_{k}} / v_{r_{k}}\left(r_{k} e_{n}\right)$ and $u_{r_{k}} / u\left(r_{k} e_{n}\right)$ and rescaling back we obtain that $v / u$ has a Hölder growth rate in the $e_{n}$ direction away from the origin. When $0 \notin \partial\{v>0\}$, we simply apply the previous argument to $-v$. Now combined with Lemma 3.14 and interior estimates we have a Hölder continuity estimate at every point including those at the boundary. In order to make the estimate uniform across any domain $D_{L}$ with $L \leq M$, we apply a compactness argument.

\section{Second-Order Elliptic Operators}

The techniques employed in Section 3 can be applied to other elliptic operators, and in this section we extend the results of Section 3 to second order linear elliptic operators in divergence form on Lipschitz domains. Specifically, we consider operators of the form

$$
\mathcal{L} u=\left(a^{i j} u_{i}\right)_{j}+b^{i} u_{i}+c u .
$$

We assume the following ellipticity conditions:

$$
\Lambda^{-1}|\xi|^{2} \leq\left\langle a^{i j}(x) \xi, \xi\right\rangle \leq \Lambda|\xi|^{2},
$$

for some $\Lambda>0$ and for all nonzero $\xi \in \mathbb{R}^{n}$. Furthermore, $a^{i j}(x)$ is a real $n \times n$ matrix. For the lower order terms we assume $|c(x)|, \sum\left|b^{i}(x)\right| \leq \Lambda-1$ and that $c(x) \leq 0$.

We will continue with the notation of Section 3, however, for now, we write $u \in \mathcal{S}_{\mathcal{L}}\left(D_{L, R}\right)$ if

$$
\begin{aligned}
\mathcal{L} u(x) & =0 \text { in } D_{L, R} \cap B_{R}, \\
u(x) & =0 \text { on } D_{L, R}^{c} \cap B_{R},
\end{aligned}
$$

and that $u \in \mathcal{S}_{\mathcal{L}}\left(D_{L, R}, d^{\gamma}\right)$ if

$$
\begin{aligned}
|\mathcal{L} u(x)| & \leq\left(\operatorname{dist}\left(x, \partial D_{L, R} \cap B_{R}\right)\right)^{\gamma} \text { in } D_{L, R}, \\
u(x) & =0 \text { on } D_{L, R}^{c} \cap B_{R} .
\end{aligned}
$$

To apply the Hölder continuity estimates for elliptic operators we will require that

$$
\gamma>-2 / n
$$

see Theorem 8.27 in [7]. Since the boundary is Lipschitz, this will ensure the correct integrability assumptions for the right hand side. We will assume these bounds throughout the section whenever referencing $\mathcal{S}_{\mathcal{L}}\left(D_{L, R}, d^{\gamma}\right)$.

From the forthcoming Lemma 4.1, it will follow that if $u \geq 0$ and $u \in$ $\mathcal{S}_{\mathcal{L}}\left(\mathcal{C}_{L}, \infty\right)$, then $u$ is unique up to multiplicative constant and we again denote $u$ by $u_{L}$; however, $u_{L}$ will not necessarily be homogeneous. We recall that $\mathcal{C}_{L}$ is defined although not convex when $L<0$. To emphasize when $-L<0$, we again write $\mathcal{C}_{(-L)}$ and $u_{(-L)}$ when $u \in \mathcal{S}_{\mathcal{L}}\left(\mathcal{C}_{(-L)}\right)$. We will follow the same outline as in Section 3. 
In Section 3 we utilized the standard boundary Harnack principle. Since the standard boundary Harnack principle is unavailable when considering the zeroorder term $c(x)$, we prove the next two Lemmas under the situation $b^{i}, c \equiv 0$.

Lemma 4.1. Let $u, v \in \mathcal{S}_{\mathcal{L}\left(D_{L, \infty}\right)}$ with $u \geq 0$. Assume also that $b^{i}, c \equiv 0$. If there exists $C>0$ such that

$$
\sup _{B_{R}}|v| \leq C \sup _{B_{R}} u \text { for } R \geq 1,
$$

then there exists $c \in \mathbb{R}$ such that $v \equiv c u$.

Proof. When $b^{i}, c \equiv 0$, there is a standard Boundary Harnack principle for divergence form equations [2]; therefore, the proof of Lemma 3.6 holds in this situation, and so the proof of Lemma 3.7 also holds as well.

Theorem 4.2. Assume $\mathcal{L}$ has no lower order terms; that is, $\mathcal{L} w=\partial_{j}\left(a^{i j} u_{i}\right)$. Let $v, u \in \mathcal{S}_{\mathcal{L}}\left(D_{L, \infty}\right)$ with $u \geq 0$, and assume $b^{i}, c \equiv 0$. If there exists $C>0$ such that

$$
|v(x)| \leq C \ln (|x|+1) u(x) \text { for }|x| \geq 1,
$$

then $v(x)=c u(x)$ for some $c \in \mathbb{R}$.

Remark 4.3. The proof given below for Theorem 4.2 depends on the function $g$ being slowly varying at $\infty$. Thus, the same proof will actually show a stronger result: If for every $\varepsilon>0$ there exists $C_{\varepsilon}$ such that

$$
|v(x)| \leq C_{\varepsilon}|x|^{\varepsilon} u(x) \text { for }|x| \geq 1,
$$

then $v=c u$ for some $c \in \mathbb{R}$.

Proof. Suppose by way of contradiction that $v$ is not a constant multiple of $u$. Then by Lemma 4.1 we have

$$
\limsup _{R \rightarrow \infty}\left(\sup _{B_{R}} \frac{|v(x)|}{u(x)}\right)=\infty
$$

If $h(R)=\sup _{B_{R}}|v| / u$, let $g$ be the concave envelope of $h$. By assumption $g(R) \leq$ $C \ln (R+1)$ for $R \geq 1$. The function $g$ satisfies,

$$
\lim _{R \rightarrow \infty} \frac{g(C R)}{g(R)}=1 \text { for any } C>0 .
$$

There also exist infinitely many $R_{k}$ such that $h\left(R_{k}\right)=g\left(R_{k}\right)$. We define

$$
u_{k}(x):=\frac{u\left(R_{k} x\right)}{\sup _{B_{R_{k}}} u} \text { and } v_{k}(x)=\frac{v\left(R_{k} x\right)}{\sup _{B_{R_{k}}}|v|} .
$$

From (4.1) we have that for any fixed $\rho>1$, that

$$
\lim _{R_{k} \rightarrow \infty} \frac{\sup _{B_{\rho}}|v|}{\sup _{B_{\rho}} u} \leq 1 .
$$


By uniform continuity estimates up to the boundary for divergence form equations, by picking a subsequence we have that

$$
\lim _{R_{k} \rightarrow \infty} u_{k}(x)=w_{1}
$$

and that $u_{k}$ converges uniformly to $w_{1}$ on compact sets. From (4.2) we also have that

$$
\lim _{R_{k} \rightarrow \infty} v_{k}(x)=w_{2},
$$

with uniform convergence on compact sets and with $\left|w_{2}\right| \leq C w_{1}$. There also exists a limiting operator $\mathcal{L}_{0}$ and limiting Lipschitz domain $\tilde{D}$ such that $w_{1}, w_{2} \in$ $\mathcal{S}_{\mathcal{L}_{0}}\left(\tilde{D}_{L, \infty}\right)$. Then from Lemma 4.1 we conclude that $w_{2}=c w_{1}$ for some $c \in \mathbb{R}$. Without loss of generality we assume $c=1$.

Then for any $\varepsilon>0$, there exists $N_{\varepsilon} \in \mathbb{N}$ such that if $k \geq N_{\varepsilon}$, then

$$
\sup _{B_{2}}\left|v_{k}-u_{k}\right|<\varepsilon
$$

By the standard boundary Harnack principle

$$
\sup _{B_{1}} \frac{\left|v_{k}-u_{k}\right|}{u_{k}} \leq C \frac{\left\|v_{k}-u_{k}\right\|_{L^{\infty}\left(B_{2}\right)}}{u_{k}\left(e_{n} / 2\right)} \leq C \varepsilon .
$$

We fix $x^{1} \in D_{L, \infty}$. We also have

$$
\lim _{R_{k} \rightarrow \infty} \frac{\left|v_{k}\left(R_{k}^{-1} x^{1}\right)\right|}{\left|u_{k}\left(R_{k}^{-1} x^{1}\right)\right|} \leq \frac{v\left(x^{1}\right)}{u\left(x^{1}\right)} \frac{1}{g\left(R_{k}\right)} \rightarrow 0 .
$$

Then for large enough $k$ we have $\left|v_{k}\left(R_{k}^{-1} x^{1}\right)\right| \leq u_{k}\left(R_{k}^{-1} x^{1}\right) / 2$. Finally, we conclude then that

$$
\frac{u\left(x^{1}\right) / 2}{u\left(x^{1}\right)} \leq \sup _{B_{1}} \frac{\left|v_{k}-u_{k}\right|}{u_{k}} \leq C \varepsilon .
$$

The $C$ in the above estimate only depends on the ellipticity constants of $a^{i j}$ and the Lipschitz constant for the domain. Consequently, we may choose $\varepsilon$ small enough so that $2 C \varepsilon<1$, which implies $u\left(x^{1}\right)=0$ which is a contradiction since $u>0$ in $D_{L, \infty}$.

For the remainder of the section we no longer assume that the lower order terms are zero.

Lemma 4.4. Let $L \leq M$ and $u \in \mathcal{S}_{\mathcal{L}}\left(D_{L}, d^{\gamma}\right)$ with $u \geq 0$ and $\mathcal{L} u \leq 0$. Assume also $0 \in \partial D_{L}$. Let $x \in \partial D_{L} \cap B_{1 / 2}$ and let $y \in D_{L} \cap B_{r}(x)$ with $r \leq 1 / 4$. Then there exists a constant $C$ depending only on dimension $n, M$, and $\operatorname{dist}\left(y, \partial D_{L}\right)$ such that

$$
\begin{aligned}
& \sup _{B_{r}(x)} u \leq C u(y) \quad \text { for all } r \leq 1 / 4, \\
& \sup _{B_{1 / 4}} u \geq C^{-1} u(y) .
\end{aligned}
$$


Proof. Since $u \geq 0$ and $\mathcal{L} u \leq 0$ and $u \in \mathcal{S}_{\mathcal{L}}\left(D_{L, R}, d^{\gamma}\right)$, this is an application of the interior weak Harnack inequality as well as uniform Hölder continuity up to the boundary, see [7].

We now state the analogue of Lemma 3.2.

Lemma 4.5. Let $D_{L_{k}, R_{k}}$ be a sequence of domains with $L_{k} \leq M, R_{k} \geq 1$, and $0 \in \partial D_{L_{k}}$. Let $u_{k} \in \mathcal{S}_{\mathcal{L}}\left(D_{L_{k}, R_{k}}, d^{\gamma}\right)$, and assume $\gamma>-2 / n$. Further, assume either that

$$
\begin{aligned}
& \text { (1) } u_{k} \geq 0 \text { and } \sup _{B_{1 / 2}} u_{k} \leq 1 \text {, or } \\
& \text { (2) } \sup _{B_{r}}\left|u_{k}\right| \leq C r^{\beta} \text { for } r \leq 1 \text { and some constants } C, \beta>0 .
\end{aligned}
$$

Then there exists a subsequence with a limiting domain $D_{L_{0}, R_{0}}$ and a limiting function $u_{0} \in \mathcal{S}\left(D_{L_{0}, R_{0}}, d^{\gamma}\right)$ such that

$$
\sup _{B_{r}}\left|u_{k}-u_{0}\right| \rightarrow 0
$$

for all $r<R_{0}$.

Proof. This is an application of uniform Hölder continuity up to the boundary, see [7].

Lemma 4.6. Let $u \in \mathcal{S}_{\mathcal{L}}\left(D_{L}\right)$ with $u \geq 0$ and $\mathcal{L} u \leq 0$. Let $L<M$. There exist constants $c_{1}, c_{2}, \beta, \alpha$ depending only on $n, M, \Lambda$ and $M-L$ such that for any $x \in \partial D_{L} \cap B_{1 / 2}$,

$$
\begin{aligned}
& \text { (1) } \sup _{B_{r}(x)} u \geq c_{1} u\left(e_{n} / 2\right) r^{\alpha}, \\
& \text { (2) } \sup _{B_{r}(x)} u \leq c_{2} u\left(e_{n} / 2\right) r^{\beta},
\end{aligned}
$$

for any $r \leq 1 / 4$.

Proof. Property (2) is just uniform Hölder continuity up to the boundary. Assume by way of contradiction that (1) is not true. Then there exists a sequence satisfying

(1) $\quad u_{k} \in \mathcal{S}_{\mathcal{L}_{k}}\left(D_{L_{k}}, d^{\gamma}\right)$

(2) $u_{k} \geq 0$

(3) $\mathcal{L}_{k} u_{k} \leq 0$

(4) $\quad u_{k}\left(e_{n} / 4\right) \leq \frac{u_{k}\left(e_{n} / 2\right)}{k} \quad$ from Lemma 4.4 .

We rescale and let

$$
w_{k}=\frac{u_{k}\left(r_{k} x\right)}{\sup _{B_{r_{k}}} u_{k}} .
$$

From Lemma 4.5 we have that $w_{k} \rightarrow w_{0}$ uniformly and there is a limiting Lipschitz domain $D_{L_{0}}$ and limiting elliptic operator $\mathcal{L}_{0}$ such that $u \in \mathcal{S}_{\mathcal{L}_{0}}\left(D_{L_{0}}\right)$. Now $w_{0} \geq 0$, and from the definition of $w_{k}$ we conclude that $w_{0}$ is not identically zero. However, $w_{0}\left(e_{n} / 4\right)=0$, which contradicts the strong maximum principle. 
With the previous result, the proof of Theorem 4.7 proceeds exactly as in the case of Theorem 3.10.

Theorem 4.7. Let $0 \in \partial D_{L}$ with $L<M$, and fix $x^{0} \in D_{L}$. Assume further that $B_{1} \cap\left\{x_{n}>1 / 4\right\} \subseteq D_{L}$. Let $\gamma>-2 / n$ and let $\alpha$ be as in Lemma 4.6. Assume $u, v \geq 0$ and $u\left(x^{0}\right)=v\left(x^{0}\right)=1$, and also assume that $2-\alpha+\gamma>0$ with $\alpha$ as given in Lemma 4.6. Then there exists a uniform constants $C, \varepsilon>0$ (depending only on dimension $n$, Lipschitz constant $M, M-L$, and $\left.\operatorname{dist}\left(x^{0}, \partial D_{L}\right)\right)$ such that if

$$
-\left(\operatorname{dist}\left(x, \partial\left(D \cap B_{1}\right)\right)\right)^{\gamma} \leq \mathcal{L} u, \mathcal{L} v \leq \varepsilon\left(\operatorname{dist}\left(x, \partial\left(D \cap B_{1}\right)\right)\right)^{\gamma}
$$

then

$$
C^{-1} v(x) \leq u(x) \leq C v(x)
$$

for all $x \in B_{1 / 2}$.

Proof. The proof proceeds exactly as the proof of Theorem 3.10. We only highlight how the lower order terms vanish in the blow-up regime. The rescaled functions

$$
w_{r, k}(x):=\frac{v_{k}(r x)-\frac{v_{k}\left(r y_{k}\right)}{u_{k}\left(r y_{k}\right)} u_{k}(r x)}{\sup _{B_{1} \cap \mathcal{C}}\left|v_{k}(r x)-\frac{v_{k}\left(r y_{k}\right)}{u_{k}\left(r y_{k}\right)} u_{k}(r x)\right|},
$$

satisfy

$$
\mathcal{L}_{r} w_{r, k}=\frac{r^{2} \mathcal{L} v_{k}(r x)}{\sup _{B_{1} \cap \mathcal{C}}\left|v_{k}(r x)-\frac{v_{k}\left(r y_{k}\right)}{u_{k}\left(r y_{k}\right)} u_{k}(r x)\right|},
$$

where for a function $f$ we have

$$
\mathcal{L}_{r} f=\left(a^{i j}(r x) f_{i}\right)_{j}+r b^{i}(r x) f_{i}+r^{2} c(r x) f .
$$

Thus, in the blow-up regime the lower order terms disappear. As in the proof of Theorems 2.1 and 3.10 we may bound the denominator in (4.4) from below by $r_{k}^{\alpha}\left[\ln \left(1 / r_{k}\right)\right]^{2}$ for the constructed sequence of $r_{k} \rightarrow 0$. Using that the numerator is bounded from above by $r_{k}^{2-\gamma}$, we have that in the blow-up regime the right hand side and lower order terms vanish. We then apply Lemma 4.1 and Theorem 4.2 as in the proof of Theorem 3.10.

Acknowledgements. Open access funding provided by Royal Institute of Technology. The authors thank the referee for helpful suggestions, such as that to include the case when the right hand side is positive but small.

Open Access This article is distributed under the terms of the Creative Commons Attribution 4.0 International License (http://creativecommons.org/licenses/by/4.0/), which permits unrestricted use, distribution, and reproduction in any medium, provided you give appropriate credit to the original author(s) and the source, provide a link to the Creative Commons license, and indicate if changes were made.

Publisher's Note Springer Nature remains neutral with regard to jurisdictional claims in published maps and institutional affiliations. 


\section{Appendix A.}

Lemma A.1. Let $\left\{a_{k}\right\}$ be a sequence satisfying $a_{k} \geq 0$ and $\sum_{k=1}^{\infty} a_{k}=+\infty$. Then there exists a subsequence $a_{k_{l}}$ such that for any $j \in \mathbb{N}$,

$$
\limsup _{k_{l} \rightarrow \infty} \frac{\sum_{i=1}^{j} a_{k_{l}-i}}{a_{k_{l}}} \leq j .
$$

Proof. If $\lim \sup a_{k}=\infty$, one may simply choose a subsequence $a_{k_{l}}$ such that $a_{k_{l}-i} \leq a_{k_{l}}$ for any $0<i<k_{l}$ and the result immediately follows. If $0<\lim \sup a_{k}<\infty$, one may choose a subsequence $a_{k_{l}}$ such that $\lim a_{k_{l}}=\lim \sup a_{k}$, and the result also follows.

We now consider the most difficult case when $\lim \sup a_{k}=0$. Define $f_{1}(k)=a_{k}$ for $k \in \mathbb{N}$, and interpolate linearly between integers for any value $x \geq 1$. Note that $\lim _{x \rightarrow \infty} f_{1}(x)=0$. Let $k_{1}=\max \left\{j \in \mathbb{N} \mid a_{j}=\max \left\{a_{k}\right\}\right\}$. We inductively choose

$$
k_{l+1}=\max \left\{j>k_{l} \mid a_{j}=\max \left\{a_{k}\right\}_{k=k_{l}+1}^{\infty}\right\} .
$$

We define $f_{2}\left(k_{l}\right)=a_{k_{l}}$ and interpolate linearly in between values of the subsequence $\left\{k_{l}\right\}$. Then $f_{2}(x)$ is strictly decreasing, and $f_{2}(k) \geq a_{k}$ for $k \geq k_{1}$.

We now use an inductive procedure to construct $f_{3}(x)$ which will be strictly decreasing and convex. We choose a further subsequence by letting $k_{l_{1}}=k_{1}$. If $k_{l_{i}}$ has been chosen, then we define $f_{3}$ by letting $f_{3}\left(k_{l_{j}}\right)=a_{k_{l_{j}}}$ for $1 \leq j \leq i$ and linearly interpolating for all other values $k_{l_{1}} \leq x \leq k_{l_{i}}$. We choose

$$
k_{l_{i+1}}=\min \left\{k_{j} \mid k_{j}>k_{l_{i}} \text { and } \frac{f_{3}\left(k_{l_{i}}-1\right)-f_{3}\left(k_{l_{i}}\right)}{2}+\frac{f_{2}\left(k_{j}\right)-f_{2}\left(k_{l_{i}}\right)}{2\left(k_{j}-k_{l_{i}}\right)} \geq 0\right\} .
$$

Such a minimum will exist since $f_{3}(x)$ is strictly decreasing on $k_{l_{1}} \leq x \leq k_{l_{i}}$. Then $f_{1}(x) \leq f_{2}(x) \leq f_{3}(x)$, and $f_{3}(x)$ is strictly decreasing, convex, and $f_{3}\left(k_{l_{i}}\right)=a_{k_{l_{i}}}$. For convenience we relabel $f_{3}(x)=g(x)$ and relabel our subsequence $k_{l_{i}}$ to be $k_{l}$.

Since $g>0$, decreasing, and convex we may take a smooth approximation $g_{\varepsilon}(x)$ such that $g_{\varepsilon} \geq 0, g_{\varepsilon}^{\prime} \leq 0$, and $g_{\varepsilon}^{\prime \prime} \geq 0$ it follows that $g_{\varepsilon}^{\prime}(x+1) g_{\varepsilon}(x) \leq g_{\varepsilon}^{\prime}(x) g_{\varepsilon}(x) \leq g_{\varepsilon}^{\prime}(x) g_{\varepsilon}(x+1)$ so that

$$
\frac{d}{d x}\left[\frac{g_{\varepsilon}(x+1)}{g_{\varepsilon}(x)}\right] \leq 0 .
$$

Then $g(x+1) / g(x)$ is a decreasing function, so that $\lim _{x \rightarrow \infty} g(x+1) / g(x)$ exists.

Since $g(k) \geq a_{k}$ and the series $\sum a_{k}$ diverges, then the series $\sum g(k)$ diverges. By the ratio test it follows that

$$
\lim _{k \rightarrow \infty} \frac{g(k-1)}{g(k)} \leq 1 .
$$

This proves

$$
\lim _{k \rightarrow \infty} \frac{\sum_{i=1}^{j} g(k-i)}{g(k)} \leq j,
$$

when $j=1$. By induction, we assume that thus holds true for $j$. Then

$$
\begin{aligned}
\lim _{k \rightarrow \infty} \frac{\sum_{i=1}^{j+1} g(k-i)}{g(k)} & =\lim _{k \rightarrow \infty} \frac{g(k-j+1)}{g(k)}+\frac{\sum_{i=1}^{j} g(k-i)}{g(k)} \\
& =\lim _{k \rightarrow \infty} \prod_{i=1}^{j+1} \frac{g(k-i)}{g(k-(i-1))}+\frac{\sum_{i=1}^{j} g(k-i)}{g(k)},
\end{aligned}
$$


and by (A.2) as well as the induction hypothesis, we conclude that

$$
\lim _{k \rightarrow \infty} \frac{\sum_{i=1}^{j+1} g(k-i)}{g(k)} \leq 1+j .
$$

Finally, we use that $a_{k_{l}}=g\left(k_{l}\right)$ and $a_{k_{l}-i} \leq g\left(k_{l}-i\right)$ to conclude (A.1).

\title{
References
}

1. Athanasopoulos, I., Caffarelli, L.A.: A theorem of real analysis and its application to free boundary problems. Commun. Pure Appl. Math. 38(5), 499-502, 1985

2. Caffarelli, L., Fabes, E., Mortola, S., SAlsa, S.: Boundary behavior of nonnegative solutions of elliptic operators in divergence form. Indiana Univ. Math. J.30(4), 621-640, 1981

3. Caffarelli, L.A.: The obstacle problem revisited. J. Fourier Anal. Appl. 4(4-5), 383402, 1998

4. Caffarelli, L., Salsa, S.: A Geometric Approach to Free Boundary Problems, Graduate Studies in Mathematics, vol. 68. American Mathematical Society, Providence, RI 2005

5. De Silva, D., Savin, O.: A note on higher regularity boundary Harnack inequality. Discrete Contin. Dyn. Syst. 35(12), 6155-6163, 2015

6. Evans, L.C.: Partial differential equations, second ed., Graduate Studies in Mathematics, vol. 19, American Mathematical Society, Providence, RI, 2010

7. Gilbarg, D., Trudinger, N.S.: Elliptic partial differential equations of second order, Classics in Mathematics, Springer-Verlag, Berlin, 2001, Reprint of the 1998 edition

8. Monneau, R., Weiss, G.S.: An unstable elliptic free boundary problem arising in solid combustion. Duke Math. J. 136(2), 321-341, 2007

9. Petrosyan, A., Shahgholian, H., Uraltseva, N.: Regularity of Free Boundaries in Obstacle-Type Problems, Graduate Studies in Mathematics, vol. 136. American Mathematical Society, Providence, RI 2012

10. Shahgholian, H.: When does the free boundary enter into corner points of the fixed boundary?.Zap. Nauchn. Sem. S.-Peterburg. Otdel. Mat. Inst. Steklov. (POMI) 3102004 , no. Kraev. Zadachi Mat. Fiz. i Smezh. Vopr. Teor. Funkts. 35 [34], 213-225, 229

11. SweErs, G.: Hopf's lemma and two-dimensional domains with corners. Rend. Istit. Mat. Univ. Trieste 28 (1996) (suppl.), 383-419 (1997), Dedicated to the memory of Pierre Grisvard

\author{
Mark Allen \\ Department of Mathematics, \\ Brigham Young University, \\ Provo \\ UT \\ 84602 USA. \\ and \\ Henrik Shahgholian \\ Department of Mathematics, \\ KTH Royal Institute of Technology, \\ Stockholm, \\ Sweden. \\ e-mail: henriksh@math.kth.se
}

(Received December 11, 2018 / Accepted June 26, 2019)

Published online July 10, 2019

(C) The Author(s) (2019) 